This item was submitted to Loughborough's Research Repository by the author.

Items in Figshare are protected by copyright, with all rights reserved, unless otherwise indicated.

\title{
Low-frequency wake dynamics for a square-back vehicle with side trailing edge tapers
}

PLEASE CITE THE PUBLISHED VERSION

https://doi.org/10.1016/j.jweia.2018.12.009

\section{PUBLISHER}

(C) Elsevier BV

\section{VERSION}

AM (Accepted Manuscript)

\section{PUBLISHER STATEMENT}

This paper was accepted for publication in the journal Journal of Wind Engineering and Industrial Aerodynamics and the definitive published version is available at https://doi.org/10.1016/j.jweia.2018.12.009

\section{LICENCE}

CC BY-NC-ND 4.0

\section{REPOSITORY RECORD}

Pavia, Giancarlo, Martin Passmore, and Max Varney. 2019. "Low-frequency Wake Dynamics for a Squareback Vehicle with Side Trailing Edge Tapers”. figshare. https://hdl.handle.net/2134/36533. 


\title{
Low-frequency wake dynamics for a square-back vehicle with side trailing edge tapers
}

\author{
Giancarlo Pavia ${ }^{a}$, Martin Passmore, Max Varney \\ Stewart Miller Building, Loughborough University, Loughborough, Leicestershire LE11 3TU, United Kingdom \\ ${ }^{a}$ G.Pavia@lboro.ac.uk
}

\begin{abstract}
In this paper, the effects of side trailing edge tapering on the wake of a simplified square-back vehicle are investigated. The tapered surfaces are reported to trigger a switch from a laterally asymmetric bi-stable wake to a vertically asymmetric stable wake. The wake structure reported in the literature for lateral symmetry breaking states is seen to rotate by $90^{\circ}$ as the angle of the tapered surfaces $\phi_{s}$ is increased. A $6 \%$ drag reduction over the simple square-back case is reported for $6^{\circ}<\phi_{s}<12^{\circ}$. This gain is found to be the result of the stretching of the circular vortex responsible for the suction zone visible in any symmetry breaking state. A downwash dominated wake is observed in these conditions. The sensitivity of such a wake to small variations of the model pitch angle (for $\phi_{s}=12^{\circ}$ ) is also assessed. As the pitch angle $\alpha$ is reduced from $0^{\circ}$ to $-2^{\circ}$, the time averaged wake is reported to switch from a downwash dominated topology to an upwash dominated topology. A strengthening of the long-time instability is observed when the symmetry in the vertical direction is recovered and is accompanied with a $4.9 \%$ reduction in base drag over the same model tested at $\alpha=0^{\circ}$.
\end{abstract}

Keywords: Bluff body aerodynamics, Wake dynamics, Wake bi-stability

\section{Introduction}

Since the seminal work of Grandemange et al. (2013b), the long-time dynamic behaviour of the wakes developing downstream of square-back bluff bodies has been the object of an ever-growing interest in the scientific community. Over the last few years, remarkable progress has been made in the characterisation (Volpe et al. (2015), Pavia et al. (2018)), modelling (Brackston et al. 2016) and control (Grandemange et al. (2014b), Evrard et al. (2016)) of such instability. Evidence of the presence of this unsteady behaviour has been found also on geometries more relevant to the transport industry (Grandemange et al. (2015) and Pavia and Passmore (2017)) and commercially available passenger cars (Bonnavion et al., 2017), making it of great interest for the engineering community.

Strong analogies have been seen between this mode and the multi-stable behaviour reported for the wake developing downstream of axisymmetric geometries (Rigas et al. 2014). In the latter case, the symmetry breaking mode has been shown to be highly sensitive to small variations of the angle between the body's axis of revolution and the onset flow (Wolf and Stumpf (2014) and Gentile et al. (2017)) as well as perturbations applied to either the boundary layer developing around the body or the shear layer bounding the wake (Grandemange et al. (2012), Mariotti (2018)).

Focusing on the effects of base aspect ratio on the bi-stable mode, Grandemange et al. (2013a) reported a bi-stable motion for the wake of a square-back body in the lateral direction for $W>H$ and in the vertical direction when $W<H$. An 'interfering region', where the vertical and lateral reflectional symmetry breaking modes may coexist, was in fact isolated for
$0.77 \lesssim W / H \lesssim 1.30$, at a non dimensional ground clearance $C^{*}>0.08$ (where $C^{*}=h / H$, with $h$ referring to the distance between the bottom flat surface of the model and the ground). Within this range, it was postulated that instabilities from both the shortest and the largest side may occur, although the limit case at $W / H=1$ was not observed in the experiment, arguably due to the presence of residual asymmetries in the experimental setup.

For a square-back geometry with $W>H$, a strong relationship has been found between the time averaged wake topology in the mid-vertical plane and the tendency of the wake to develop a bi-stable behaviour in the lateral direction. Perry et al. (2016), pointed out that the lateral bi-stable behaviour was weakened in the case of either upwash- or downwashdominated wakes as well as when the distance separating the top and bottom shear layer was reduced while preserving the symmetry of the time averaged wake in the vertical direction. Barros et al. (2017), perturbing the flow passing underneath a square-back Ahmed body before separation, showed that the restoration of the flow symmetry along a plane normal to the ground was always accompanied with the occurrence of a bistable motion of the wake in the spanwise direction, revealing the existence of a bifurcation scenario where the flow switches between a wall-normal asymmetric recirculation and a bi-stable spanwise configuration. During the transition between these two scenarios, meandering motions of the wake between asymmetric wall-normal and spanwise states were also reported, although without the presence of a perfect bimodal distribution.

In Grandemange et al. (2013a), when a configuration with $W / H=0.75$ was tested at different values of the ground clearance $C^{*}$, a bi-stable behaviour was seen only for $C^{*}=0.10$ 
and $0.50 \leq C^{*} \leq 1.00$, whereas a stable state, asymmetric in the vertical direction, was reported for $0.1<C^{*}<0.5$, with $\partial C_{p} / \partial z^{*}<0$ over the model base. A similar asymmetry was described by Cabitza (2014) when considering a prismatic model with $W / H=0.86$, tested at a ground clearance of $C^{*}=0.56$. In that case, the author speculated that the cause of the asymmetry was to be found in the proximity of the model with the ground. Nevertheless, when the shear layers were perturbed using synthetic jets and the sensitivity of the system to different forcing frequencies and amplitudes was tested, a bi-stable behaviour was seen along both vertical and spanwise directions. Interestingly, the control strategy yielding the lowest drag was found to stabilise the wake in a non-symmetric configuration, aligned with one of the two diagonals of the base.

Similar results in terms of time averaged base pressure distribution were reported by Perry (2016), testing a square-back model at $C^{*} \approx 0.2$, with $W / H \approx 1$ and a leading edge radius of $r / W \approx 0.10$. The time averaged velocity field along the model centreline showed strong similarities with that seen in the mid-horizontal plane obtained for lateral symmetry breaking states (Volpe et al., 2015): it features a circular vortex acting close to the upper portion of the base and an elliptical vortical structure forming along the bottom shear layer, in proximity to the wake closure. For the same geometry, Perry (2016) reported the appearance of a bi-stable motion, mostly along the lateral direction, when the boundary layer developing along the lateral surfaces was perturbed by changing the ride height $\left(0.13 \lesssim C^{*} \lesssim 0.33\right)$ and/or altering the shape of the model front-end. A comparable topology was reported by Van Raemdonck and Van Tooren (2008), when testing a prismatic model with $W / H=0.74$ at $C^{*}=0.14$. However, no information was provided on the wake dynamics. Traces of asymmetry in the vertical direction had been previously reported for the wake developing downstream of a square-back Ahmed body with $W / H=1$ by Krajnovic and Davidson (2003), performing LES simulations. The asymmetry, however, was found to almost completely disappear when the level of refinement of the mesh was increased, further suggesting the existence of a strong relationship between the boundary layer development and the wake's size and topology. A stable vertical asymmetry can also be seen in the experimental results collected by McArthur et al. (2016). In this case, although the model employed had strong similarities with that considered by Van Raemdonck and Van Tooren (2008), the topology of the wake along the model centreline was shown to be a mirror image of that described in the previous cases. One explanation for this may be found in the different shape of the model front-end, featuring an upper leading edge with a radius $(r / W=2.35)$ almost 20 times larger than radii used for the side and lower leading edges $(r / W=0.12)$. Nevertheless, the short-time wake dynamics described in Volpe et al. (2015) and Pavia et al. (2018) were still recognisable, with a pumping motion occurring at $S t_{H} \approx 0.08$ and a lateral flapping seen at $S t_{H} \approx 0.17$. For a similar geometry, Castelain et al. (2018), reported a switch from an upwash dominated stable wake to a downwash dominated stable wake when the underbody blockage was increased from $\approx 0 \%$ to $\approx 90 \%$.

Forcing the lateral shear layers has been found to be an ef- fective way to stabilise the wake (Brackston et al. (2016), Pavia et al. (2016)), while also reducing drag. In particular, Pavia et al. (2016) pointed out that the application of high aspect ratio tapers to the vertical trailing edges of the Windsor body stabilised the wake in a vertically asymmetric state. In these conditions, an improvement of up to $13 \%$ was seen in the pressure recovery over the model rear facing surfaces. A similar gain in terms of drag reduction had already been reported for the same configuration by Perry et al. (2015), although with a different time averaged base pressure distribution. When compared, the time averaged pressure maps presented in Pavia et al. (2016) and Perry et al. (2015) are mirror images, although no evident differences can be found in the experimental setup between the two cases.

The present work aims to shed some light on the mechanism promoting the stabilisation of the wake in such conditions while also investigating the cause of the discrepancies reported in the literature. For this reason, an experimental campaign consisting of balance measurements, pressure tappings and PIV acquisitions was carried out in the Loughborough University Large Wind Tunnel, using the same model as in Perry et al. (2015) and Pavia et al. (2016), equipped with side edge tapers with chamfer angles $\phi_{s}$ up to $20^{\circ}$. For the model with $\phi_{s}=12^{\circ}$, the sensitivity of the wake to variations of the pitch angle $\alpha$ (up to $\left.-2^{\circ}\right)$ is also assessed.

\section{Experimental Methodology}

\subsection{The Windsor Body.}

The Windsor body employed in the work reported here is as used in Perry et al. (2016) and Pavia et al. (2018) (Fig. 1a). It is a simplified geometry featuring a slanted front-end, forming a $23^{\circ}$ angle with the flat roof. Rounded leading edges, with radii of $50 \mathrm{~mm}$ at the nose and $200 \mathrm{~mm}$ on the roof, are also employed, to avoid flow separation. These features make this model more representative of mass produced passenger cars than the Ahmed model (Ahmed et al., 1984), used by Grandemange et al. (2013b), Volpe et al. (2015) and Evrard et al. (2016)). At the scale used in this work (length $L=1044 \mathrm{~mm}$, width $W=389 \mathrm{~mm}$, height $H=289 \mathrm{~mm}$ ) the Windsor model is approximately equivalent to a $1 / 4$ scale passenger car. A removable rear section has also been used, allowing the testing of multiple rear-end shapes.

Different side trailing edge tapers have been investigated, following a similar approach to that of Perry et al. (2015) and Pavia et al. (2016) (Fig. 1a). Each taper has a chord $c$ of $45 \mathrm{~mm}$, giving a fixed aspect ratio of $A R=6.4$ (with $A R$ defined as the ratio between the height of the model and the chord of the taper); slant angles $\phi_{s}$ of $0^{\circ}, 6^{\circ}, 12^{\circ}, 16^{\circ}$, and $20^{\circ}$ have been considered, with $\phi_{s}$ denoting the angle formed between each vertical taper and the model's sides. The sensitivity of the wake's topology and unsteady behaviour to small changes of the model pitch angle has also been investigated.

When installed in the wind tunnel, the model was connected to the six component balance located beneath the working section floor via four pins, consisting of $M 8$ threaded bars. During 


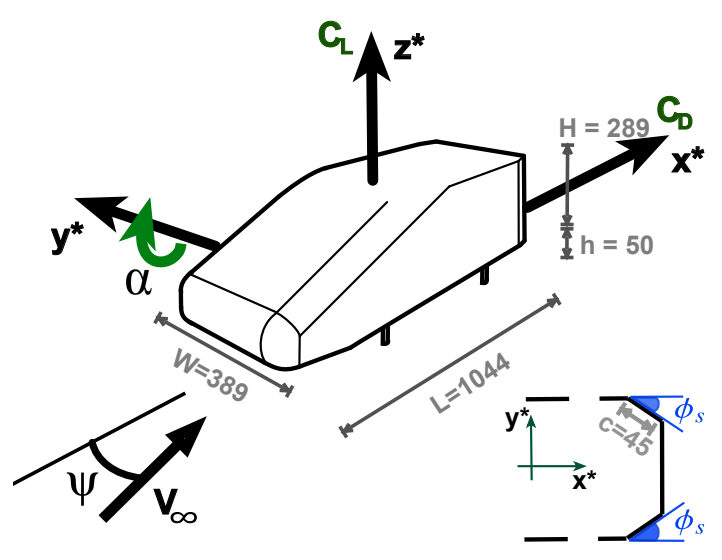

(a)

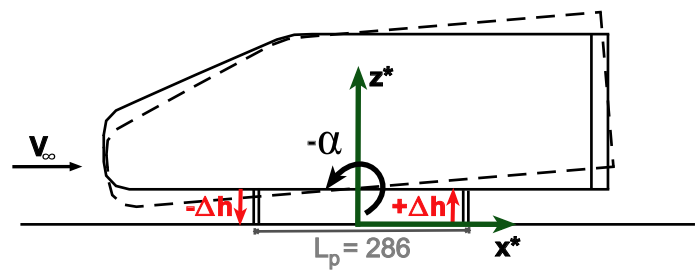

(b)

Figure 1: Representation of the model considered in the present work: $\mathbf{a}$ model with no wheels and side trailing edge tapers; $\mathbf{b}$ schematic representation of the pitch angle variation. All dimension are expressed in $\mathrm{mm}$.

the tests at zero pitch angle $\alpha$, the ground clearance was set at $50 \mathrm{~mm}\left(C^{*}=0.173\right.$ when normalised with the model height $)$. The pitch angle was changed by varying the distance between the model and the ground using the pins connecting the model with the underfloor balance. Only negative values of $\alpha$ have been considered (model pitched 'nose down'). The fore-body was lowered by $\Delta h$, with $\Delta h=\left(L_{p} / 2\right) \tan (\alpha)$ (where $L_{p}$ denotes the distance separating front and rear pins), whilst the aft-body was raised by the same amount, as depicted in Fig. 1b A digital inclinometer was used to check the inclination of the model relative to the ground, with an accuracy of $\pm 0.2^{\circ}$.

The SAE coordinate system (SAE, 2010) is used throughout; the $X$ axis is aligned with the flow in the downstream direction, the $Z$ axis is vertical, positive upwards, and the $Y$ axis follows a right handed coordinate system. The origin is on the ground plane at mid wheelbase $\left(L_{p} / 2\right)$, mid track $(W / 2)$. All the dimensions as well as the coordinates in the reference systems have been normalised using the model height $H$ as reference length and are denoted with the superscript $*$ throughout the paper. For the sake of clarity, the symbol "-" is used to indicate all the time averaged quantities whilst " " denotes conditionally averaged quantities.

\subsection{The Wind Tunnel.}

All experiments were carried out in the Loughborough University Large Wind Tunnel, (Johl, 2010), at a freestream velocity of $V_{\infty}=40 \mathrm{~m} / \mathrm{s}$ (corresponding to a Reynolds Number $R e_{H}$ of $7.7 \times 10^{5}$, based on the model height).

The tunnel features a $1.92 \mathrm{~m} \times 1.32 \mathrm{~m} \times 3.6 \mathrm{~m}\left(\mathrm{~W}_{T} \times H_{T} \times L_{T}\right)$ working section, with a fixed floor and no upstream boundary layer treatment. In empty conditions, the freestream turbulence level inside the test section is approximately $0.2 \%$, with a flow uniformity of $\pm 0.4 \%$ of the mean flow value. In this state, the boundary layer thickness at the model origin is $\delta_{99}=64 \mathrm{~mm}$.

\subsection{Balance Measurements.}

The aerodynamic loads were recorded by means of an Aerotech ${ }^{\circledR}$ six-component virtual centre balance, located under the working section of the tunnel. It features analogue to digital conversion at the load cell to minimise signal degradation, and an automated yaw mechanism with a positional accuracy of $\pm 0.1^{\circ}$. Further information can be found in Johl (2010). The aerodynamic loads were sampled at $100 \mathrm{~Hz}$ for $630 \mathrm{~s}$ (corresponding to $8.720 \cdot 10^{4}$ convective units $t^{*}$, with $t^{*}=t \cdot V_{\infty} / H$ ). Before starting to $\log$ the data, a $30 s\left(t^{*}=4.152 \cdot 10^{3}\right)$ settling time was used for all measurements. All forces have been normalised using Eq. 11.

$$
C_{F i}=\frac{\text { Force }}{0.5 \rho S V_{\infty}^{2}}
$$

where $\rho$ is the air density, $S$ is the projected model frontal area $\left(S=0.1124 \mathrm{~m}^{2}\right), V_{\infty}$ is the freestream velocity. All coefficients have been corrected for blockage effects using Eq. 2

$$
C_{F i_{C o r}}=C_{F i}(1-B)^{2}
$$

where $B$ denotes the blockage value (given by the ratio between the model frontal area $S$ and the tunnel working section cross sectional area $S_{T}$ ), equal to $4.4 \%$ in the present case. For the sake of simplicity, only the longitudinal and the vertical component of the aerodynamic force have been considered in the present study (Fig. 1a).

\subsection{Pressure Measurements.}

The pressure acting over the base and the tapers was recorded by populating the rearward facing surfaces with a grid of pressure taps connected via flexible urethane tubes (with a length of $550 \mathrm{~mm}$ ) to a pair of Chell ${ }^{\circledR}$ CANdaq miniature pressure scanners (with a manufacturer quoted accuracy of $\pm 1.47 \mathrm{~Pa}$ ), 


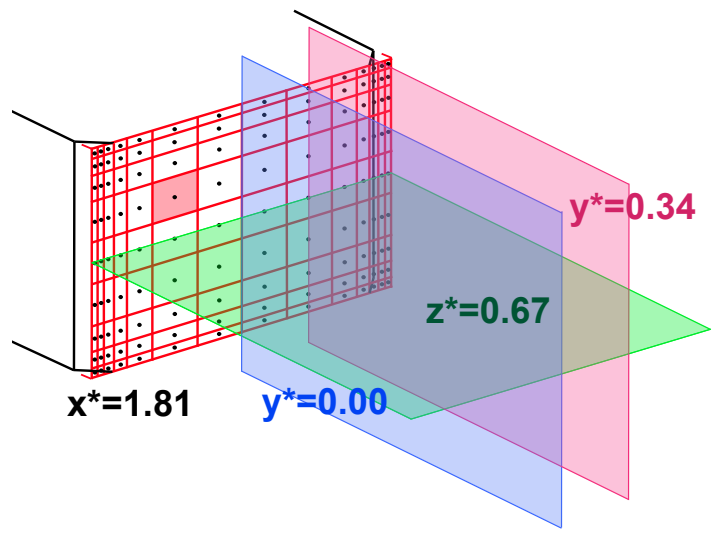

(a)

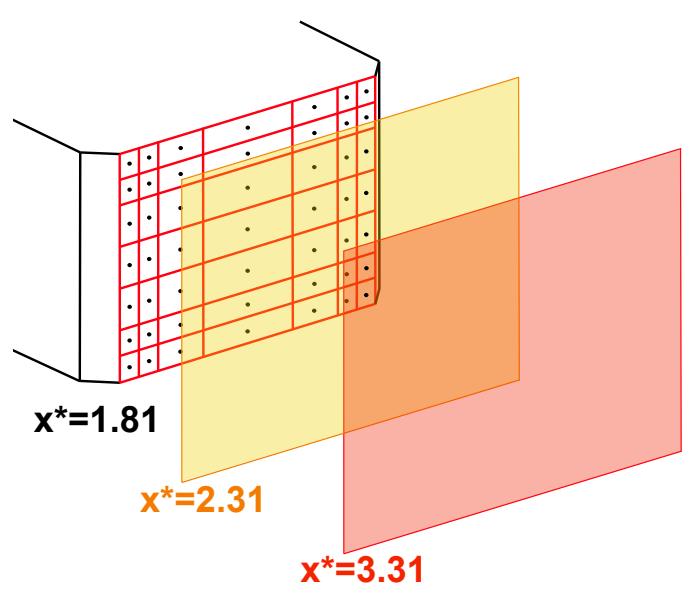

(b)

Figure 2: Representation of the model base with the locations of the pressure taps and PIV planes considered in the present work: a two scanner arrangement combined with the locations of the 2D-2C PIV planes; b single scanner arrangement combined with the locations of the 2D-3C PIV cross-planes. The base is located at $x^{*}=1.81$ from the origin of the reference system defined in Fig. 1 The red square on the model base denotes the area associated with the $i^{t h}$ tap used for the estimation for the area weighted drag.

mounted inside the model. Each scanner features 64 piezoresistive pressure sensors, paired to temperature sensors to allow for the correction of inaccuracies introduced by temperature drift. Up to 126 taps were used, depending on the configuration. The taps were placed with a finer distribution close to the edges of the model and on the tapered surfaces, to get a more accurate representation of the pressure distribution in the regions with the highest gradients (see Fig. 2a). In some cases the pressure distribution along the model centreline was also measured.

Pressure data was recorded at $260 \mathrm{~Hz}$ for $630 \mathrm{~s}$ (or $t^{*}=$ $8.720 \cdot 10^{4}$ ). The free-stream dynamic and static pressures were acquired using a Pitot-static tube mounted at the start of the test section, $1.87 \mathrm{~m}$ (or 6.47 times the model height $H$ ) upstream of the model, $100 \mathrm{~mm}$ beneath the tunnel roof. Pressure signals were corrected for magnitude and phase distortions caused by the tubing applying the correction already used in Wood (2015) and based on the method proposed by Sims-Williams and Dominy (1998). Once the pressure coefficients were calculated according to Eq. 3 ,

$$
C_{p_{i}}=\frac{p_{i}-p_{\infty}}{0.5 \rho S V_{\infty}^{2}}
$$

where $p_{\infty}$ is the freestream static pressure, the results were corrected for blockage using the MIRA correction (based on continuity) using Eq. 4 .

$$
1-C_{p_{i_{c o r r}}}=\left(1-C_{p_{i}}\right)(1-B)^{2} .
$$

The pressure drag associated with the rearward facing surfaces was then estimated by integrating the measured pressure field:

$$
\bar{C}_{D_{\text {Rear }}}=-\frac{1}{S} \iint_{S} \bar{C}_{p} \cdot d S \simeq-\frac{1}{S} \sum_{i=1}^{N_{\text {tap }}} \bar{C}_{p_{i}} S_{i},
$$

where $\bar{C}_{p_{i}}$ is the time averaged value of the pressure coefficient recorded by the $i^{\text {th }}$ tap and $S_{i}$ is the projection of the area associated with the same tap on a plane orthogonal to the direction of the onset flow (see Fig. 2).

Since the flow field analysed in the present study is highly sensitive to any asymmetry present in the experimental setup, the model was yawed to the onset flow until the most symmetric base pressure distribution was achieved, following a similar procedure to that adopted by Evrard et al.(2016) and Pavia et al. (2018). The resulting value of $\Psi$ was assumed to be where the model axis and onset flow axis were aligned.

The regions with the highest level of unsteadiness were localised considering the distribution of the root mean square of the pressure fluctuation $\left(R M S\left(\Delta C_{p}\right)\right)$ over the model base, calculated as follow:

$$
\operatorname{RMS}\left(\Delta C_{p_{i}}\right)=\sqrt{\frac{\sum_{n=1}^{N_{t}}\left(C_{p_{i}}^{n}-\bar{C}_{p_{i}}\right)^{2}}{N_{t}}}
$$

where $C_{p_{i}}^{n}$ denotes the $n^{\text {th }}$ value recorded in time by the $i^{\text {th }}$ tap and $N_{t}$ indicates the number of samples in time.

Proper Orthogonal Decomposition (POD) was used to isolate the main features of the unsteady pressure field, following the same approach proposed by Lumley (1967). A generic 
dataset $\mathbf{F}(\mathbf{x}, \mathbf{t})$ is decomposed as:

$$
\mathbf{F}(\mathbf{x}, t)=\mathbf{F}_{0}(\mathbf{x})+\mathbf{f}(\mathbf{x}, t)=\mathbf{F}_{0}(\mathbf{x})+\sum_{n=1}^{N_{t}} \boldsymbol{\Phi}^{n}(\mathbf{x}) a_{n}(t),
$$

where $N_{t}$ indicates the number of measurement points in time, $\mathbf{F}_{0}$ the mean of the considered field and $\mathbf{f}(\mathbf{x}, t)$ its fluctuating components. The basis functions $\boldsymbol{\Phi}^{n}(\mathbf{x})$ are the so called ' $P O D$ modes' and are defined as the eigenfunctions of the covariance matrix $\mathbf{R}\left(\mathbf{x}, \mathbf{x}^{\prime}\right)$ :

$$
\mathbf{R}\left(\mathbf{x}, \mathbf{x}^{\prime}\right)=\sum_{n=1}^{N_{t}} \mathbf{f}\left(\mathbf{x}, t_{n}\right) \cdot \mathbf{f}^{T}\left(\mathbf{x}^{\prime}, t_{n}\right)=\mathbf{X} \cdot \mathbf{X}^{T},
$$

where $\mathbf{X}$ is a matrix with

$$
\mathbf{X}=\left[\mathbf{f}\left(\mathbf{x}, t_{1}\right), \mathbf{f}\left(\mathbf{x}, t_{2}\right), \ldots, \mathbf{f}\left(\mathbf{x}, t_{n}\right)\right] \in \mathbf{R}^{N_{s} \times N_{t}}
$$

and $N_{s}$ refers to the number of points sampled in space. The basis functions $\boldsymbol{\Phi}^{n}(\mathbf{x})$ can therefore be determined by solving the eigenvalue problem:

$$
\mathbf{R} \boldsymbol{\Phi}^{n}=\lambda^{n} \boldsymbol{\Phi}^{n}, \quad \boldsymbol{\Phi}^{n} \in \mathbf{R}^{N_{s}}
$$

with $\lambda^{1} \geq \ldots \geq \lambda^{n}$. The eigenvalues $\lambda^{n}$ associated with the POD modes are representative of the energy content $E$ of the fluctuations captured by each mode. A low order model can then be written as:

$$
\mathbf{F}(\mathbf{x}, t) \simeq \mathbf{F}_{0}(\mathbf{x})+\sum_{n=1}^{M} \boldsymbol{\Phi}^{n}(\mathbf{x}) a_{n}(t),
$$

with $M<N_{t}$ and the temporal coefficients $a_{n}(t)$ determined projecting each spatial mode $\boldsymbol{\Phi}^{n}$ on the original dataset $\mathbf{f}(\mathbf{x}, t)$ :

$$
a_{n}(t)=\left\langle\left(\boldsymbol{\Phi}^{n}(\mathbf{x})\right)^{T}, \mathbf{f}(\mathbf{x}, t)\right\rangle .
$$

A limitation of POD is that it does not allow to separate the frequencies of different unsteady modes, as the spectrum of the POD temporal coefficients usually contains more than one peak (Taira et al. 2017). Additional information on the frequency content of such modes was collected by performing coherence analysis between signals recorded by taps placed in different locations along the trailing edges of the model, following the approach proposed by Duell and George (1999) and already used in Pavia et al. (2018). The magnitude of the coherence between two generic synchronised signals $i$ and $j$ was calculated according to the equation:

$$
\boldsymbol{\Lambda}_{\mathbf{i j}}(f)=\frac{\left|\mathbf{P}_{\mathbf{i j}}(f)\right|^{2}}{\mathbf{P}_{\mathbf{i i}}(f) \cdot \mathbf{P}_{\mathbf{j j}}(f)},
$$

where $\mathbf{P}_{\mathbf{i j}}(f)$ is the cross-spectrum and $\mathbf{P}_{\mathbf{i i}}(f)$ and $\mathbf{P}_{\mathbf{j} \mathbf{j}}(f)$ are the auto-spectra of the signals. The phase relationship between the two signals was then determined as:

$$
\chi_{\mathbf{i j}}(f)=\left|\tan ^{-1}\left(\frac{\operatorname{Im}\left(\mathbf{P}_{\mathbf{i j}}(f)\right)}{\operatorname{Re}\left(\mathbf{P}_{\mathbf{i j}}(f)\right)}\right)\right|,
$$

considering for simplicity the absolute value of the phase angle.

For the sake of simplicity, most of the unsteady analysis was performed considering only the data recorded over the model base (i.e. the vertical rear surface excluding the slants). As a consequence, just one scanner was used, rather than the two scanner setup needed for estimating $\bar{C}_{D_{\text {Rear }}}$. Spatial resolution was in this case a less stringent requirement as there was no longer the need to accurately resolve the strong pressure gradients developing over the slanted surfaces. 49 taps were employed in this case, arranged as shown in Fig. 2b. The drag estimated in this case using Eq. 57refers to the base only and it will be indicated as 'base drag' $\left(C_{D_{\text {Base }}}\right)$.

\subsection{PIV Measurements.}

$2 D-2 C$ PIV fields were taken on three orthogonal planes. Two vertical and one horizontal streamwise planes were considered (Fig. 2a), using a setup similar to that described in Perry (2016). The first vertical plane $\left(y^{*}=0\right)$ was placed along the model centreline whilst the second plane $\left(y^{*}=0.34\right)$ was located at $1 / 4$ of the model width (on the right-hand side of the model). In a similar way, the horizontal plane $\left(z^{*}=0.67\right)$ was placed at the middle of the model base. Stereoscopic PIV measurements were also performed, on planes orthogonal to the onset flow, at two streamwise locations, $x^{*}=2.31$ and $x^{*}=3.31$ (Fig. 2b). These locations are the same used in Pavia et al. (2018) and were chosen because they correspond approximately to $1 / 3$ of the bubble length and the end of the recirculating region.

LaVision ${ }^{\circledR}$ cameras were used during each acquisition. Two Imager Pro $X 4 M$ dual frame cameras, with a resolution of $2048 \times 2048$ pixels and a pixel size of $7.4 \mu \mathrm{m}^{2}$, were employed for the vertical planes. Two CMOS cameras, with a resolution of $2560 \times 2160$ pixels and a pixel size of $6.5 \mu \mathrm{m}^{2}$ were instead used for the horizontal plane as well as the two $2 D-3 C$ crossplanes. Each camera was equipped with a Nikon ${ }^{\circledR}$ Nikkor lens, with a focal length of $50 \mathrm{~mm}$; the aperture was set at $f_{\#}=5.6$ or $f_{\#}=4$, depending on the experiment. For the $2 D-2 C$ acquisitions, the cameras were placed next to each other, giving an $\approx 800 \times 400 \mathrm{~mm}\left(L_{F} \times H_{F}\right)$ field of view, with a $\approx 50 \mathrm{~mm}$ overlapping region. Optical access for the cameras was provided by the wind tunnel glass side walls, for the vertical planes, and a perspex window mounted on the wind tunnel roof, for the horizontal case. For the cross-planes the cameras were mounted on a pair of aluminium rails placed inside the tunnel working section, following the arrangement proposed by Wood (2015). The separation angle between the cameras was $\approx 50^{\circ}$. A tilt system was mounted between the lenses and the main body of the cameras in this case, in order to satisfy the Scheimplfug criterion (Prasad, 2000). The resultant field of view $\left(W_{f} \times H_{f}\right)$ was $520 \times 400 \mathrm{~mm}$.

An acquisition time of $137.7 \mathrm{~s}$ or $t^{*}=1.906 \cdot 10^{4}$ was considered in all cases. 1000 image pairs were captured for the $2 D-2 C$ planes, at a rate of $7.26 \mathrm{~Hz} .2000$ image pairs were recorded for $2 D-3 C$ cross-planes, with an acquisition rate of $15.00 \mathrm{~Hz}$. The data was then processed using DaVis 8. A calibration correction based on a pinhole model was applied, resulting in all cases in a RMS of the fit between the regular 


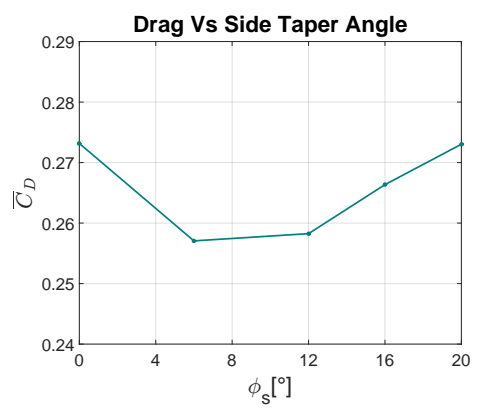

(a)

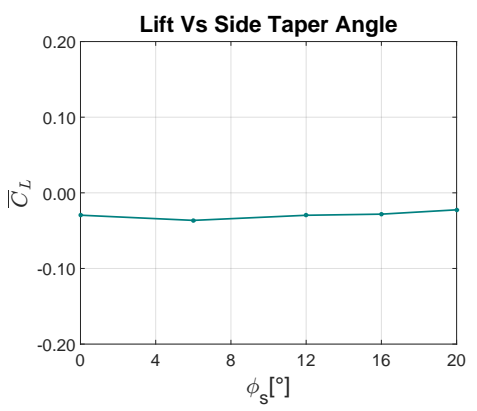

(b)

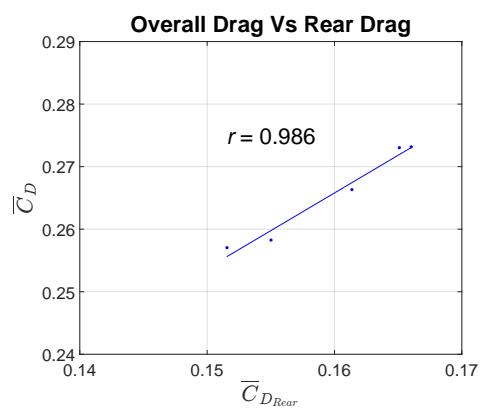

(c)

Figure 3: Time averaged drag $[\mathbf{a}$ and lift $\mathbf{b}$ ) recorded for all the configurations tested. $\mathbf{c}$ correlation between the aerodynamic drag and the pressure drag associated with the rearward facing surfaces of the model.

grid and the de-warped image of less than 0.3 pixels. Image pre-processing was performed in order to mitigate the effects of background light intensity, spurious reflections as well as image distortion. For the same reasons, the outermost regions of the field of view were discarded using a geometric mask. A multi-pass scheme for cross-correlation was applied (Willert and Gharib, 1991). The level of uncertainty associated with the measurements was estimated to be $\approx 0.4 \%$ of the mean value of the free stream velocity for the vertical planes as well as the horizontal plane and $\approx 0.5 \%$ for the $2 D-3 C$ cross-planes, having considered a 99\% confidence level (Benedict and Gould. 1996).

As for the base pressure, the main unsteady features of the velocity field were isolated by applying POD to the resulting vector fields. The snapshot method developed by Sirovich (1987) was applied in this case, due to its higher computational efficiency when the temporal domain is much smaller than the spatial domain $\left(N_{t} \ll N_{s}\right)$. This method relies on solving an eigenvalue problem of smaller size:

$$
\mathbf{X}^{T} \mathbf{X} \mathbf{A}_{n}=\lambda^{n} \mathbf{A}_{n}, \quad \mathbf{A}_{n} \in \mathbf{R}^{N_{t}}
$$

with $\mathbf{X}$ defined as in Eq. 9 The POD modes $\boldsymbol{\Phi}^{n}$ are then given by:

$$
\boldsymbol{\Phi}^{n}=\frac{1}{\sqrt{\lambda^{n}}} \mathbf{X} \mathbf{A}_{n} \in \mathbf{R}^{N_{s}}, \quad n=1,2, \ldots, N_{t},
$$

whilst the temporal coefficients $a_{n}(t)$ can still be determined using Eq. 12 .

\section{Time averaged results}

The application of tapers to the model vertical trailing edges is seen to yield a drag reduction of up to $\approx 6 \%$ over the squareback case, for chamfer angles $\phi_{s} \leq 12^{\circ}$ (Fig. 3a). This represents $\mathrm{a} \approx 2.5 \%$ improvement over the lowest drag configuration studied in Perry et al. (2016), where similar tapers were applied to the horizontal trailing edges leaving the side edges squared. $\bar{C}_{D}$ is then seen to increase for larger taper angles, until reaching a value similar to that obtained for the square-back configuration at $\phi_{s}=20^{\circ}$, in agreement with the findings reported by Perry et al. (2015) for a similar case. No particular changes, on the other hand, are observed in the time averaged values obtained for the lift force in the same conditions (Fig. 3b). A good correlation is found between the drag acting over the entire model $\bar{C}_{D}$ and the pressure drag generated by the model rearward facing surfaces $\bar{C}_{D_{\text {Rear }}}$ (calculated according Eq. 5 ) in agreement with the findings of Perry et al. (2016). This confirms the fact that the variations seen in $C_{D}$ when increasing $\phi_{s}$ are the result of changes in the pressure distribution on the model rear-end, which in turn are triggered by alterations of the time averaged wake topology, as it can be noticed by looking at the pressure maps and velocity fields presented in Fig. 4, 5, 6

When $6^{\circ}$ tapers are applied to the model vertical trailing edges, the time averaged wake changes its orientation compared to that seen for the simple square-back case described in Pavia et al. (2018), aligning parallel to one of the two diagonals of the base (Fig. 4). This is particularly visible when considering the base pressure distribution, showing a negative pressure gradient developing from the bottom-right corner to the top-left corner. A similar orientation, although with a pressure gradient of opposite sign, was reported by Cabitza (2014) for the lowest drag configuration obtained in that case by controlling the wake past a square-back body with $W / H=0.86$ using synthetic jets applied to all four trailing edges of the model, with a non dimensional actuating frequency of $S t_{H}=13.9$ and a blowing coefficient $C_{\mu}=0.168$. The genesis of this pressure distribution becomes clearer when looking at the PIV cross-plane taken at $x^{*}=2.31$. Two counter rotating vortices, similar to those reported in Pavia et al. (2018) for a lateral symmetry breaking state, are visible in this plane. Unlike that seen in Pavia et al. (2018), however, the 2D streamlines separating the two vortices are no longer aligned with the model horizontal edges but form an angle of $\approx 31^{\circ}$ with the horizontal plane. Therefore, the symmetry in the vertical direction is lost. This is visible on both vertical PIV planes considered in the present work (at $y^{*}=0.00$ and $y^{*}=0.34$ ), showing an expansion of the upper recirculation and a simultaneous shrinking of the lower vortical structure. At the same time, the distance between the centre of the vortical structures and the base of the model is also changed. The upper vortex is brought closer to the base whilst the bottom recirculation is moved further downstream.

The re-orientation of the time averaged wake topology be- 

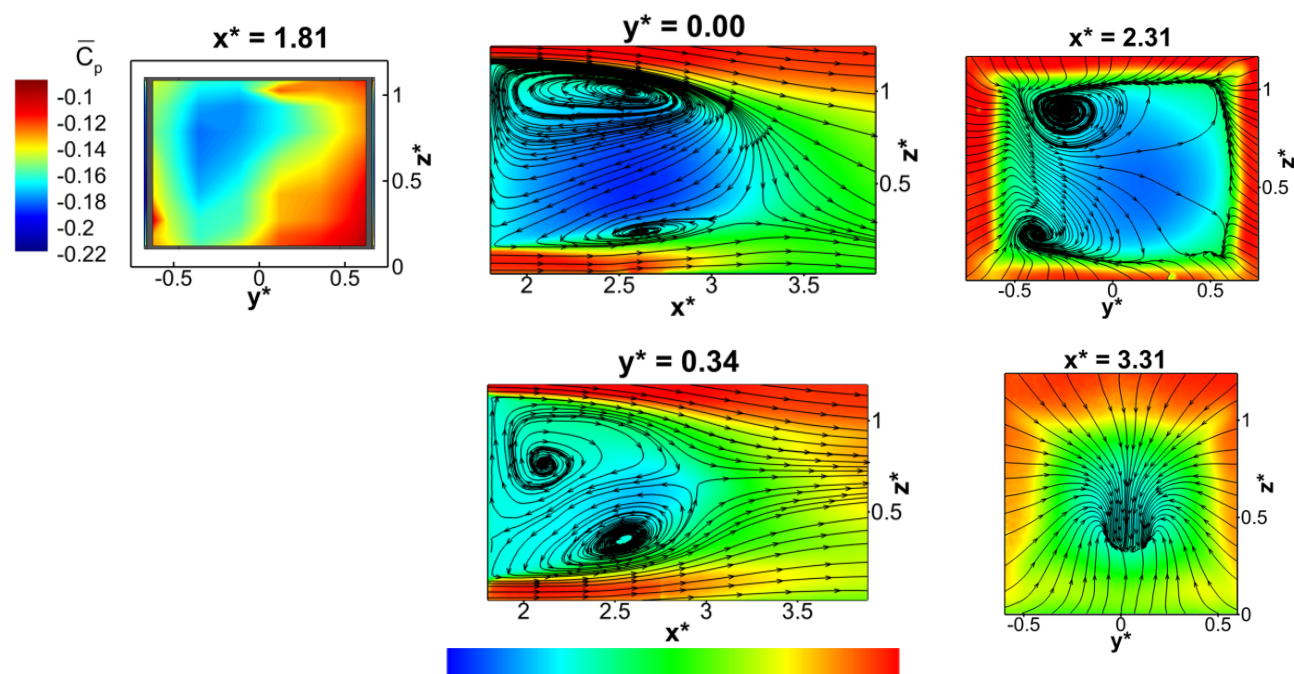

$\bar{u}^{\star}:-0.40-0.20-0.00 \quad 0.20 \quad 0.40 \quad 0.60 \quad 0.80 \quad 1.00$

Figure 4: Time averaged fields for the configuration with $\phi_{s}=6^{\circ}$. Clockwise from top left: $\mathbf{x}^{*}=\mathbf{1 . 8 1}$ base pressure distribution; $\mathbf{y}^{*}=\mathbf{0 . 0 0}$ PIV vertical mid-plane; $\mathbf{x}^{*}=2.31$ and $\mathbf{x}^{*}=3.31$ PIV stereo cross-planes; $\mathbf{y}^{*}=\mathbf{0 . 3 4}$ PIV vertical off-centre plane. All PIV fields are coloured according to the values of the axial component of the velocity $\overline{u^{*}}$; the streamlines refer to the in-plane components of the velocity.
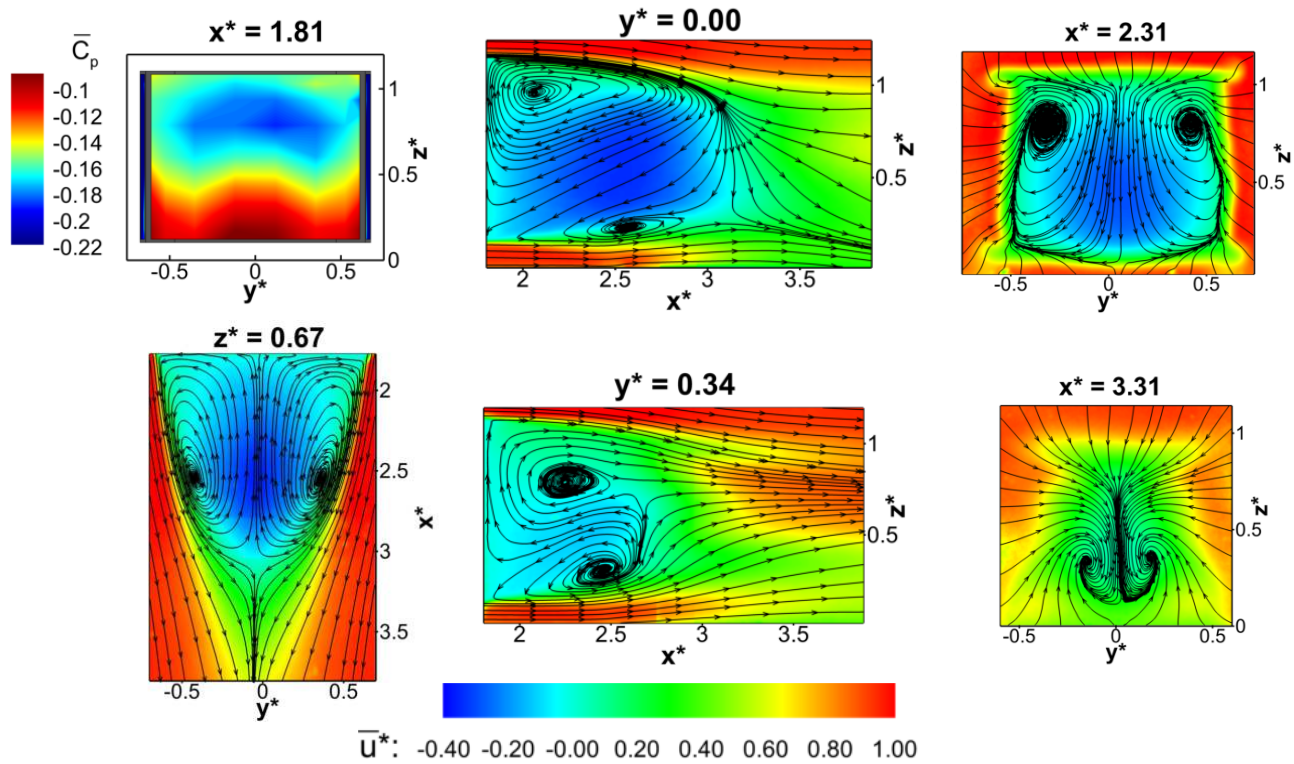

Figure 5: Time averaged fields for the configuration with $\phi_{s}=12^{\circ}$. Clockwise from top left: $\mathbf{x}^{*}=\mathbf{1 . 8 1}$ base pressure distribution; $\mathbf{y}^{*}=\mathbf{0 . 0 0}$ PIV vertical mid-plane; $\mathbf{x}^{*}=\mathbf{2 . 3 1}$ and $\mathbf{x}^{*}=3.31$ PIV stereo cross-planes; $\mathbf{y}^{*}=\mathbf{0 . 3 4}$ PIV vertical off-centre plane; $\mathbf{z}^{*}=\mathbf{0 . 6 7}$ PIV horizontal mid-plane. All PIV fields are coloured according to the values of the axial component of the velocity $\overline{u^{*}}$; the streamlines refer to the in-plane components of the velocity. 

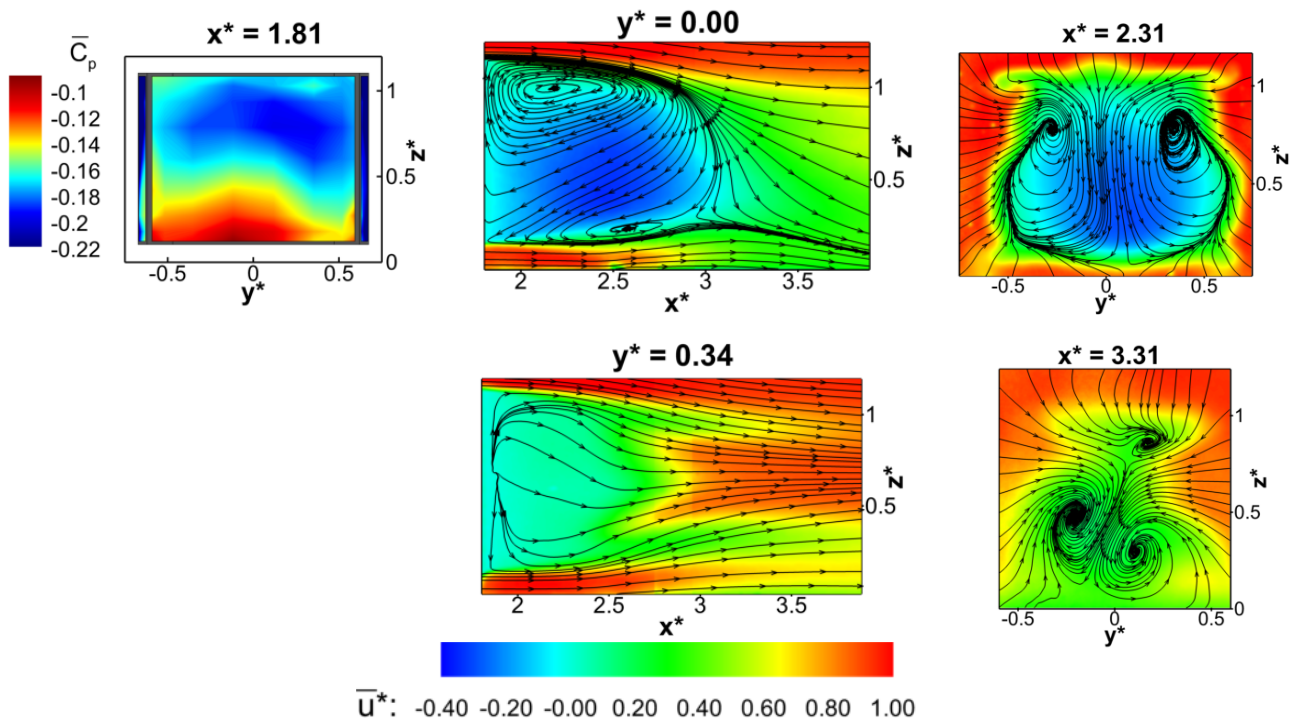

Figure 6: Time averaged fields for the configuration with $\phi_{s}=20^{\circ}$. Clockwise from top left: $\mathbf{x}^{*}=\mathbf{1 . 8 1}$ base pressure distribution; $\mathbf{y}^{*}=\mathbf{0 . 0 0}$ PIV vertical mid-plane; $\mathbf{x}^{*}=\mathbf{2 . 3 1}$ and $\mathbf{x}^{*}=\mathbf{3 . 3 1}$ PIV stereo cross-planes; $\mathbf{y}^{*}=\mathbf{0 . 3 4}$ PIV vertical off-centre plane. All PIV fields are coloured according to the values of the axial component of the velocity $\overline{u^{*}}$; the streamlines refer to the in-plane components of the velocity.

comes even more evident when the configuration with $\phi_{s}=12^{\circ}$ is considered (Fig 5). In this case, the asymmetry in the vertical direction is accentuated, but the PIV horizontal mid-plane shows that the lateral symmetry is fully restored. At this location the data shows a decrease in the width of the rear recirculation, as a consequence of the boat tailing effect of the tapered surfaces. With the loss of symmetry in the vertical direction, a negative pressure gradient develops over the base and the suction region is confined to the upper portion of the base itself, while the rear stagnation point is moved towards the bottom trailing edge. The changes in pressure distribution correlate well with the variations observed in the velocity fields captured in the two PIV cross-planes. The two counter rotating structures seen in the PIV plane at $x^{*}=2.31$ are now aligned with the top trailing edge of the model. Two additional vortical structures are also observed close to the ground in the cross-plane further downstream of the model base (at $x^{*}=3.31$ ). Overall, a downwash dominated wake is present. This may appear to contradict the trend previously described for the vertical component of the aerodynamic force (Fig. 3b), but becomes clearer when the velocity field recorded at $y^{*}=0.00$ is considered. In this location, a circular vortex can be seen forming close to the upper portion of the base whilst a smaller, elliptically shaped vortical structure can be observed in the lower portion of the wake, further downstream from the model base. This topology is consistent with the time averaged base distribution previously described. The streamlines leaving the top and bottom shear layers tend to quickly realign with the ground, resulting in a rather 'squared' wake closure. This differs from the more 'rounded' closure seen in the case of wakes associated with higher values of lift (or downforce) (Perry et al. 2016) and may explain the limited variations in terms of $C_{L}$ observed in this case.

The topology described for the plane at $y^{*}=0.00$ strongly resembles that reported by Volpe et al. (2015), (Perry et al.
2016) and Evrard et al. (2016), when characterising the lateral symmetry breaking state in a horizontal plane centred with the model base. Unlike that seen in the case of a simple squareback configuration, however, this vortical structure appears to have been 'stretched' in the streamwise direction, and in the case of the configuration with $\phi_{s}=6^{\circ}$, the distance between the core of the vortex and the model base is also increased. A similar trend is observed for the vortical structures captured in the mid-horizontal plane $\left(z^{*}=0.67\right.$ in Fig. 5). These changes in the time averaged wake topology may explain the $\approx 15 \%$ reduction in base drag $\left(\bar{C}_{D_{\text {Base }}}\right)$ obtained for $6^{\circ} \leq \phi_{s} \leq 12^{\circ}$ over the square-back case, that ultimately leads to the $\approx 6 \%$ improvement in the values recorded for both $\bar{C}_{D_{\text {Rear }}}$ and $\bar{C}_{D}$. A similar topology was reported by Perry (2016), in the case of a stable asymmetric wake, developing downstream of a simplified square-back body, with $W / H \approx 1$ and a leading edge with a radius of $r / W \approx 0.10$ (tested at $C^{*} \approx 0.2$ ). The change in shape of the larger recirculation seen in the plane at $y^{*}=0.00$, also seems to be responsible for the increase in drag reported in Fig. 3a when chamfers angles $\geq 16^{\circ}$ are applied to the model's side trailing edges. Indeed, as large chamfer angles are considered, the inflow generated by the tapered surfaces increases, yielding a shortening of the rear recirculation in the streamwise direction, but also a strengthening of the vortices developing at the tips of each slant, as a consequence of the growth of the suction acting on the tapers themselves. At the same time, the larger of the two vortical structures seen in the plane at $y^{*}=0.00$, is 'pushed' against the base of the model, assuming a rather characteristic triangular shape, thus further decreasing the pressure over this surface. This is clear in Fig. 6, when the velocity field recorded at $y^{*}=0.00$ and $y^{*}=0.34$ as well as the pressure distribution acquired at $x^{*}=1.81$ are considered. In these conditions, a progressive loss of lateral symmetry is also noted. This is seen in the base pressure map as well as the velocity field 

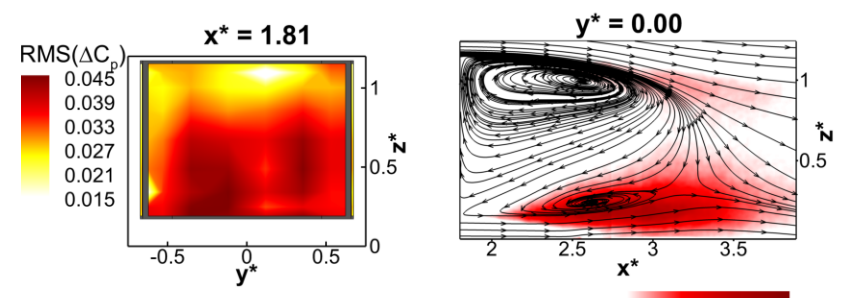

$\mathrm{K}^{*}\left(\mathrm{x} 10^{-3}\right): \quad 01020304050607080$

(a) $\phi_{s}=6^{\circ}$
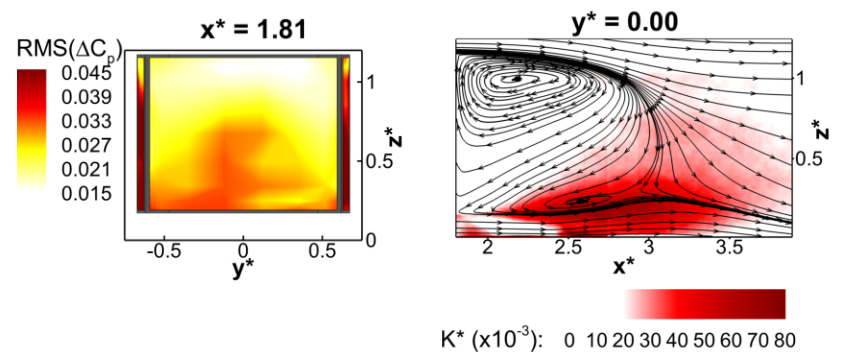

(c) $\phi_{s}=20^{\circ}$
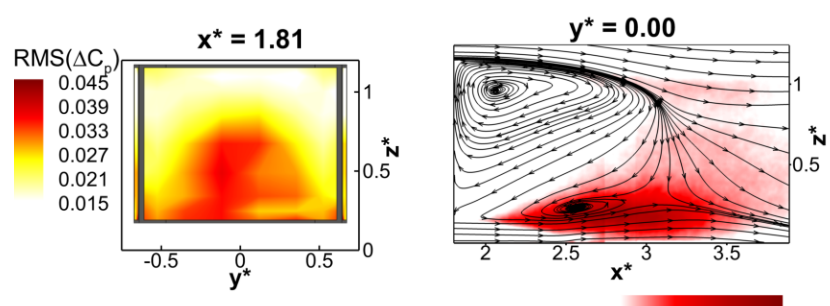

$K^{*}\left(\times 10^{-3}\right): \quad 01020304050607080$

(b) $\phi_{s}=12^{\circ}$ recorded at $x^{*}=2.31$, but becomes even more evident when the cross-plane close to the wake closure (at $x^{*}=3.31$ ) is considered, suggesting that its origin is in the shear layer instability triggered by the flow separation over the slants (see \$4) rather than in long-time motions of the flow reversal.

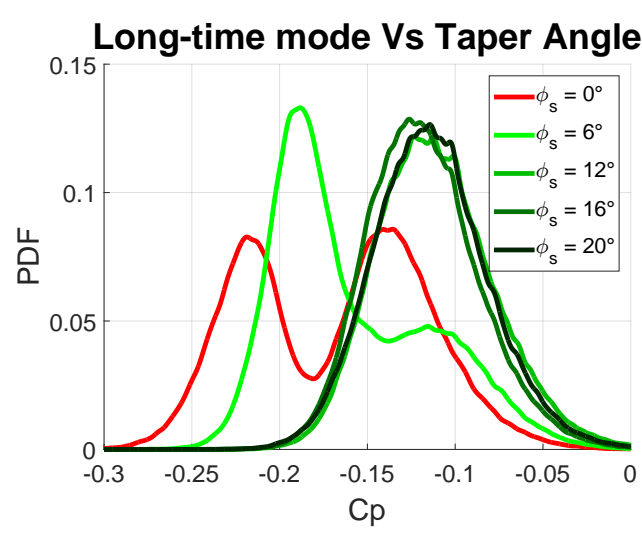

(a)

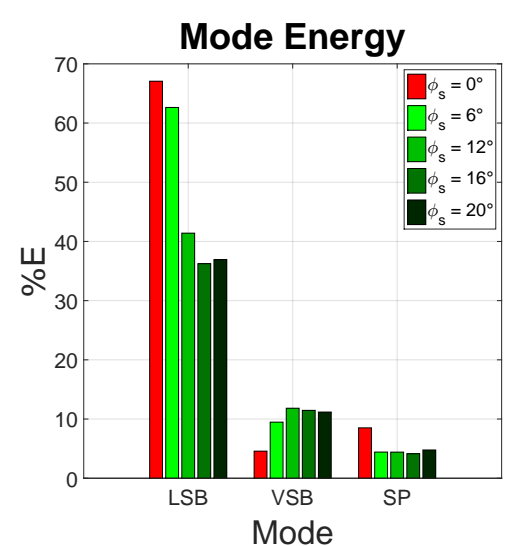

(b)

Figure 8: P Probability density function distribution (PDF) of the values of $C_{p}$ recorded by one of the pressure taps placed in the region of highest pressure fluctuation; $\mathbf{b}$ energy associated with the the lateral symmetry breaking mode (LSB), the vertical symmetry breaking mode (VSB) and the symmetry preserving mode (SP). 


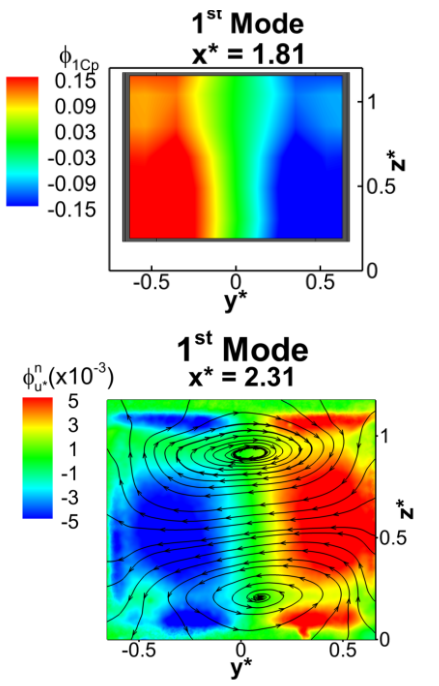

(a) LSB mode

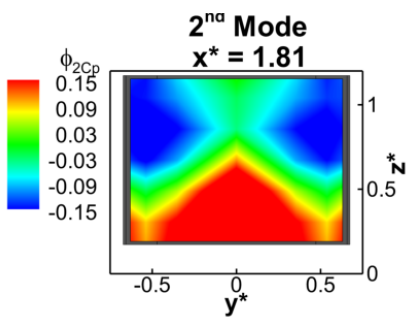

$2^{\text {nd }}$ Mode

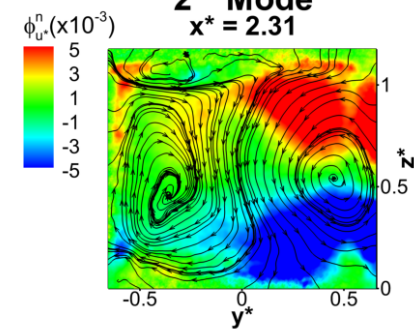

(b) VSB mode
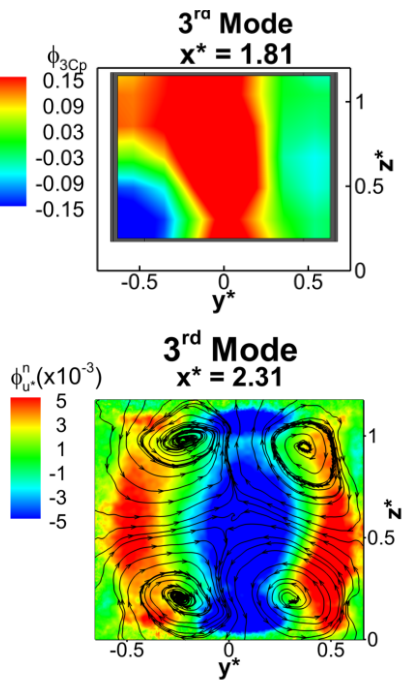

(c) SP mode

Figure 9: Spatial distribution of the POD modes extracted from the base pressure distribution (top row) and PIV cross-plane at $x^{*}=2.31$ (bottom row) for $\phi_{s}=6^{\circ}$. The modes are ordered according to their topology: a lateral symmetry breaking mode $(L S B)$, $\mathbf{b}$ vertical symmetry breaking mode $(V S B)$, $\mathbf{c}$ symmetry preserving mode $(S P) . \phi_{C_{p}}^{n}$ refers to the magnitude of the spatial eigen-modes extracted from the field of the pressure fluctuation. The eigen-functions related to the velocity

fluctuation are coloured according to the values of the through plane component $\phi_{u^{*}}^{n}$ whereas the streamlines are drawn considering the in-plane components $\phi_{v^{*}}^{n}$ and $\phi_{w^{*}}^{n}$.
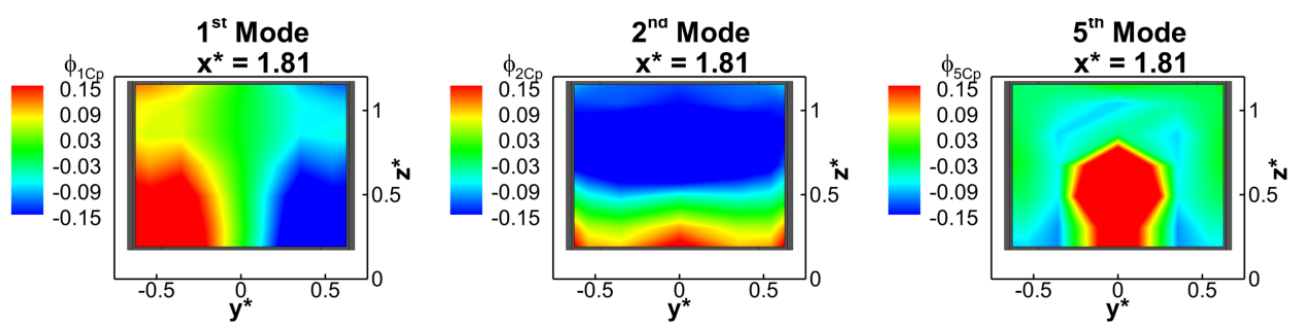

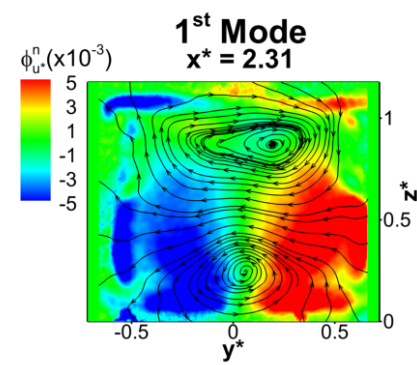

(a) LSB mode

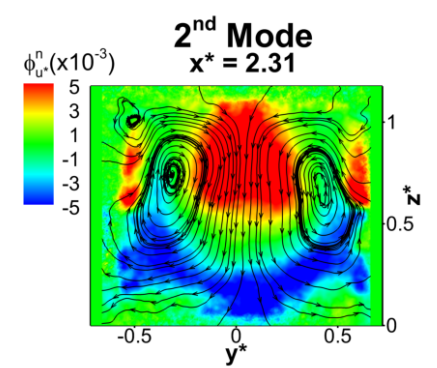

(b) VSB mode

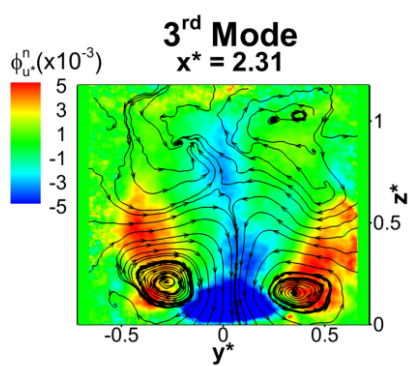

(c) SP mode

Figure 10: Spatial distribution of the POD modes extracted from the base pressure distribution (top row) and PIV cross-plane at $x^{*}=2.31$ (bottom row) for $\phi_{s}=12^{\circ}$. The modes are ordered according to their topology: a lateral symmetry breaking mode ( $\left.L S B\right)$, b vertical symmetry breaking mode $(V S B)$, $\mathbf{c}$ symmetry preserving mode $(S P) . \phi_{C_{p}}^{n}$ refers to the magnitude of the spatial eigen-modes extracted from the field of the pressure fluctuation. The eigen-functions related to the velocity fluctuation are coloured according to the values of the through plane component $\phi_{u^{*}}^{n}$ whereas the streamlines are drawn considering the in-plane components $\phi_{v^{*}}^{n}$ and $\phi_{w^{*}}^{n}$ 


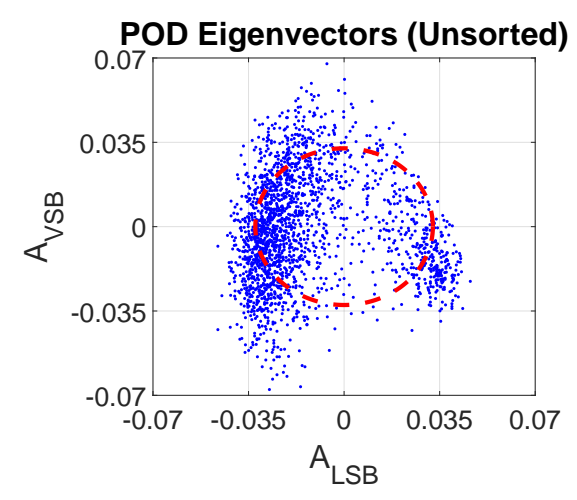

(a)

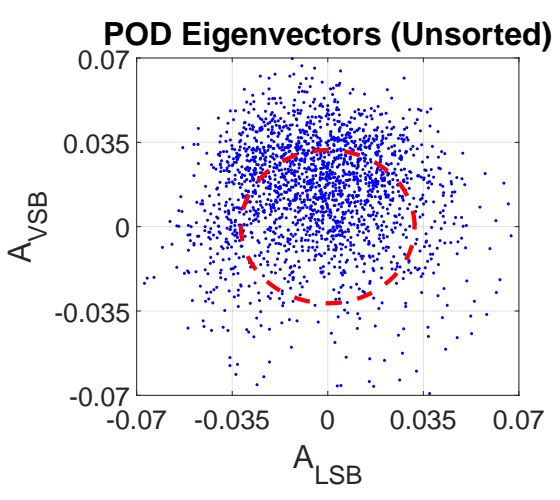

(b)

Figure 11: Scatter plot of the POD temporal coefficients associated with the $L S B$ mode and the $V S B$ mode for $\phi_{s}=6^{\circ}$ a and $\phi_{s}=12^{\circ}[\mathbf{b}$, referring to the PIV data recorded at $x^{*}=2.31$. The dashed circle represents the fitting function used for sorting the snapshots in phase (see Eq. 17).

$R M S\left(\Delta C_{p}\right)$ obtained for the square-back case, is seen to disappear, replaced (for $\phi_{s} \geq 12^{\circ}$ ) by a single region of high pressure fluctuation located in proximity to the rear stagnation point (Fig. 7b). A further reduction in the level of unsteadiness on the model base is noticed for $\phi_{s}=20^{\circ}$ (Fig. 7c). In this last case, however, a greater level of fluctuation is seen close to the tapered surfaces, as the flow fails to reattach over the slants, in analogy with that already reported by Perry et al. (2016), when studying the effects of horizontal trailing edge tapers with a similar angle. This localised increase of $R M S\left(\Delta C_{p}\right)$ may be the element causing the loss of lateral symmetry observed in $\$ 3$ in the time averaged results (Fig. 6).

Changes are also observed in the distribution of the velocity fluctuations in the near-wake region. The loss of symmetry in the vertical direction seen in the time averaged flow field is accompanied with the development of a strong disparity in the distribution of turbulent kinetic energy $K^{*}=1 / 2\left(\overline{u^{\prime * 2}}+\overline{w^{* 2}}\right)$ between the two horizontal shear layers evident in the vertical mid-planes $\left(y^{*}=0.00\right)$ reported in Fig. 7. One of the main unsteady features of the wake is represented by the interactions between the larger, stable recirculation forming downstream of the upper portion of the base and the bottom shear layer. Because of these interactions, smaller transverse vortices roll up close to the ground and are periodically shed downstream. These structures appear to be separate from the main horseshoe vortex depicted in Fig. 7d, whose existence can be inferred from the results presented in $\$ 3$. Its topology is similar to that of the 'hairpin' vortex isolated in Pavia et al. (2018) for each lateral symmetry breaking state, but with two main differences: the different orientation of the vortex, as it appears now to be rotated by $90^{\circ}$, and the presence of two streamwise vortices downstream of the wake closure rather than a single vortex.

Remarkable similarities are indeed seen between this structure and the topology reported for the vortex sheet shed from axisymmetric bodies tested at similar Reynolds number (Taneda, 1978). Similarly to that observed here, a single plane of symmetry has been reported in the near wake topology in those cases. In the absence of external perturbations, however, the plane of symmetry has been observed to randomly change orientation in the azimuthal direction with a characteristic time scale of $t \approx 5 \cdot 10^{2} D / V_{\infty}$ (Rigas et al. (2014), Gentile et al. (2016)). The selection of either one single stable position or two bi-stable sates has been reported as a consequence of the application of perturbations with azimuthal wave numbers of $m=1$ and $m=2$ respectively (Grandemange et al. (2012). Grandemange et al. (2014a), Gentile et al. (2017), Mariotti (2018)). In the case of a rectilinear body with $W>H$, the tapering of the vertical trailing edges appears to produce similar effects to those seen in the case of axisymmetric geometries subjected to a perturbation with $m=1$, although in this case the wake tends to stabilise in a plane that is orthogonal to that where the perturbation is applied.

The 'stabilisation' of the wake is highlighted by a change in the nature of the pressure fluctuations recorded over the base of the model itself. As $\phi_{s}$ is increased, the PDF of $C_{p}$ recorded by one of the pressure taps in the region of highest unsteadiness, switches from a bi-modal symmetrical shape for $\phi_{s}=0^{\circ}$ to a bi-modal non-symmetrical shape for $\phi_{s}=6^{\circ}$, with one of the two states clearly prevailing over the other. A normal distribution is eventually observed for $\phi_{s} \geq 12^{\circ}$ (Fig. 8a). In the same conditions, the energy level captured by the $1^{\text {st }}$ POD mode, dubbed 'lateral symmetry breaking mode' as it refers to motions of the wake in the lateral direction (Fig. 9a and 10a), drops by more than 20 percentage points ( $L S B$ in Fig. $8 \mathrm{~b}$ ). This drop is only marginally counterbalanced by the slight increase $(\approx 6 \%)$ observed in the energy associated with the second symmetry breaking POD mode, dubbed 'vertical symmetry breaking mode' (VS B in Fig. $8 \mathrm{~b}$ ) since it is related to motions of the wake in the vertical direction (Fig. $9 \mathrm{~b}$ and $10 \mathrm{~b}$ ). The 'stabilising' action of the tapers is also reported to trigger changes in the spatial functions associated with the same modes. Although the POD modes extracted for the configuration with $\phi_{s}=6^{\circ}$, from either the pressure dataset and the velocity field acquired at $x^{*}=2.31$ (Fig. 9), strongly resemble those described in Pavia et al. (2018) for the square-back case, the stabilisation of the upper portion of the wake seen for taper angles $\geq 12^{\circ}$ is accompanied with the suppression of all coherent motions previously observed in the same region of the flow field. Indeed, only the lower half of the wake is seen to 'move' in this case 

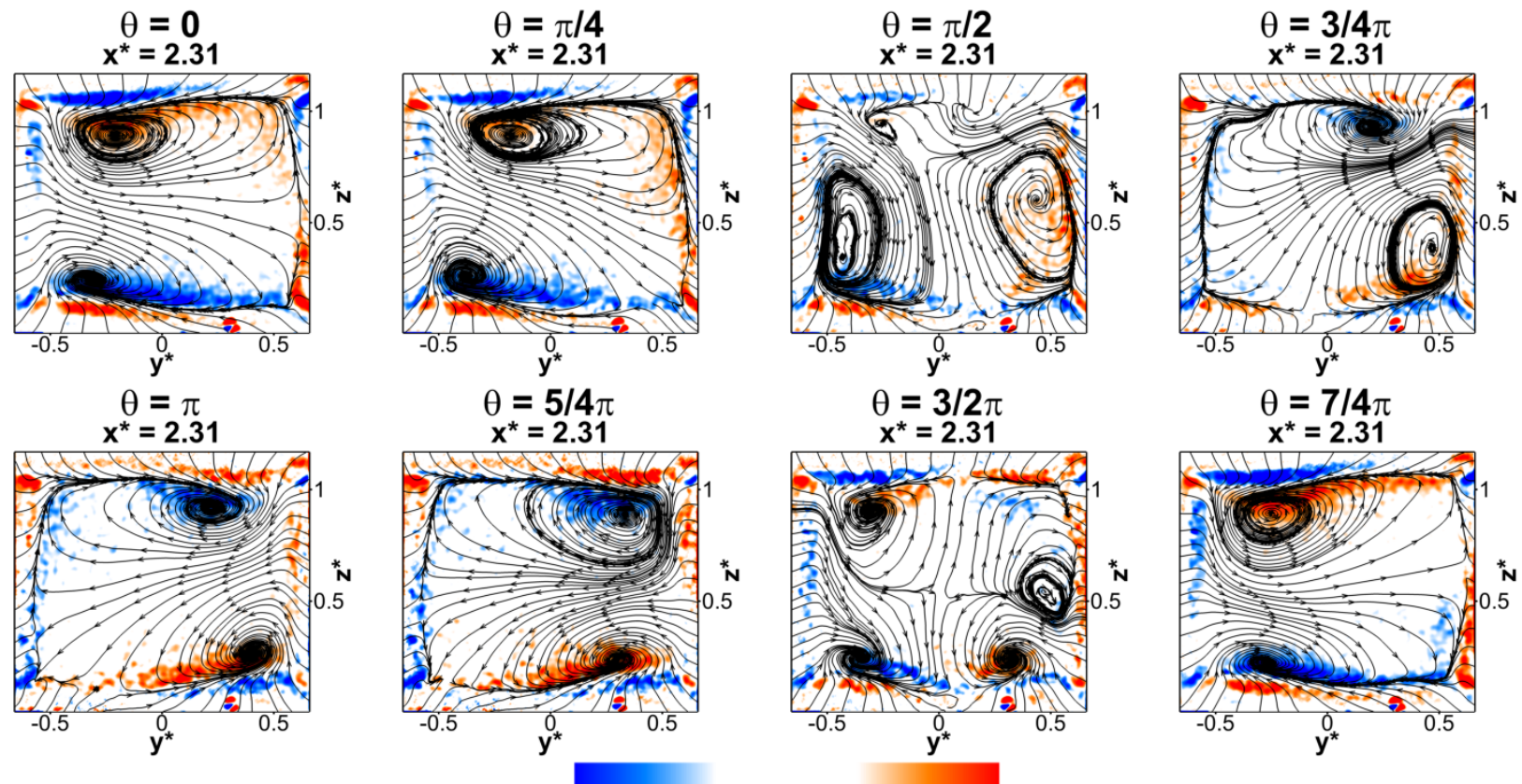

$\tilde{\Omega}_{\mathrm{x}}^{*}: \quad-2.0-1.5-1.0-0.5 \quad 0.0 \quad 0.5 \quad 1.0 \quad 1.5 \quad 2.0$

Figure 12: Low order phase averaged velocity field at $x^{*}=2.31$ for $\phi_{s}=6^{\circ}$. The plots are coloured according to the values of the normalised streamwise component of the vorticity $\tilde{\Omega}_{x}^{*}$; the streamlines are drawn considering the in-plane components of the velocity field.
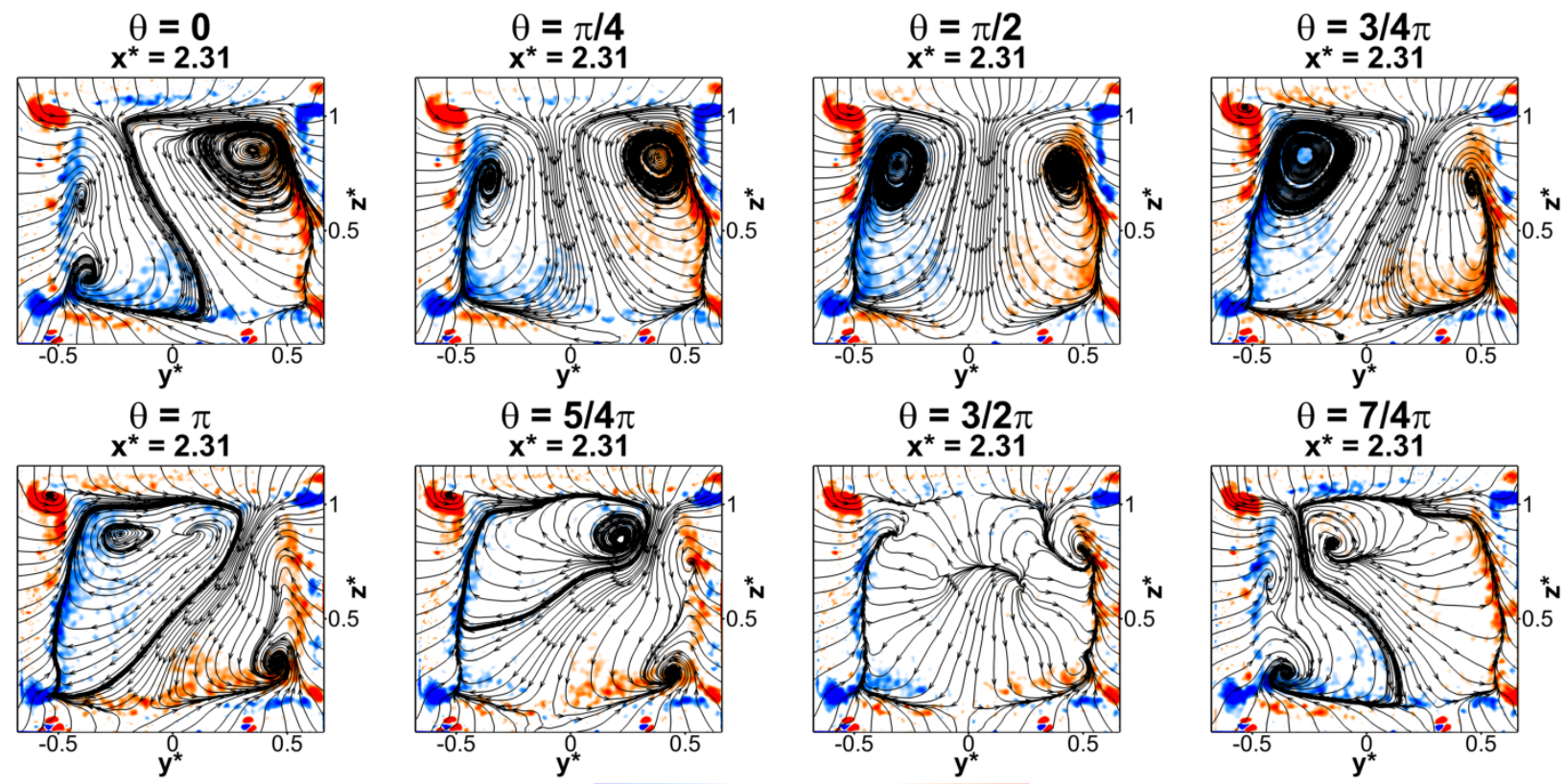

$\tilde{\Omega}_{\mathrm{x}}^{*}: \quad-2.0-1.5-1.0-0.5 \quad 0.0 \quad 0.5 \quad 1.0 \quad 1.5 \quad 2.0$

Figure 13: Low order phase averaged velocity field at $x^{*}=2.31$ for $\phi_{s}=12^{\circ}$. The plots are coloured according to the values of the normalised streamwise component of the vorticity $\tilde{\Omega}_{x}^{*}$; the streamlines are drawn considering the in-plane components of the velocity field. 


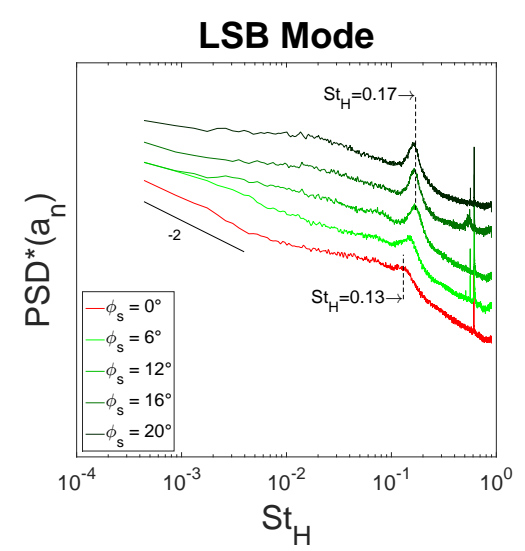

(a)

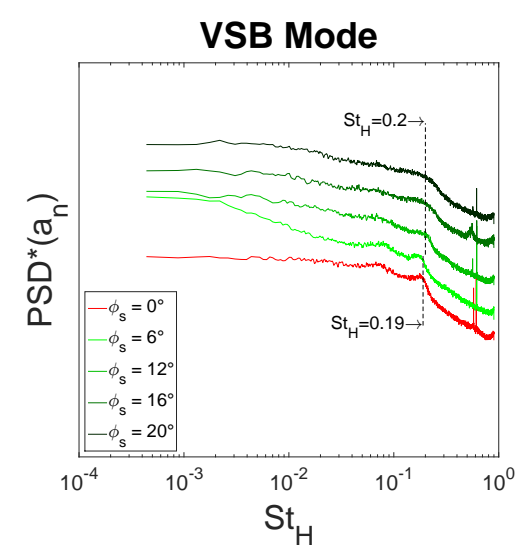

(b)

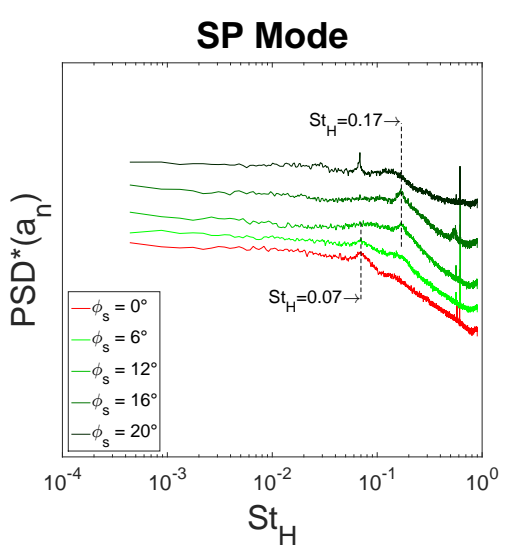

(c)

Figure 14: Spectra of the POD temporal coefficients associated with $\mathbf{a}$ the lateral symmetry breaking mode (LSB); $\mathbf{b}$ the vertical symmetry breaking mode (VSB); c the symmetry preserving mode (SP). The curves have been shifted along the vertical axis. In $\mathbf{a}$ the -2 slope seen at very low frequency for the square-back configuration is consistent with the findings of Grandemange (2013).

(Fig. 10, further confirming that seen in Fig. 7. Even bigger differences over the square-back configuration are reported when the symmetry preserving mode is considered ( $S P$ in Fig. 10). This mode has been defined in Pavia et al. (2018) as the mode that preserves the planes of symmetry characteristic of the time averaged flow field. The spatial functions presented in Fig. $10 \mathrm{c}$ show that the horizontal plane of symmetry disappears following the loss of symmetry in the vertical direction discussed in $\$ 3$ for the time averaged flow field, so that only the lateral symmetry is preserved. This is accompanied with a $\approx 50 \%$ reduction in the energy content $(E)$ compared to the same mode extracted from pressure dataset related to the square-back configuration (Fig. 8b), which explains also the lower position of the $S P$ mode in the POD energy ranking (from $2^{\text {nd }}$ in Pavia et al. (2018) to $5^{\text {th }}$ ). Indeed, the fluctuating energy captured by the $S P$ mode in this case is lower than that associated with the wind tunnel acoustic resonance (Baden Fuller. 2012) and one of the harmonics of the first mode (Rigas et al., 2014). The slight asymmetry in the lateral direction seen in Fig. 9 for the shape obtained for this mode at $x^{*}=1.81$, as well as that observed for the $V S B$ mode at $x^{*}=2.31$, is likely to be the result of small misalignments in the experimental setup (Evrard et al., 2016) that was not possible to remove within the tolerances of the instrumentation available.

Additional information can be gathered from the scatter plots between the eigenvectors $A_{V S B}$ and $A_{L S B}$ presented in Fig.11 (determined from the dataset recorded at $x^{*}=2.31$, according to Eq. 15. Following the approach proposed in Pavia et al. (2018), $A_{V S B}(t)$ and $A_{L S B}(t)$ were fitted with a pair of trigonometric functions such that the temporal coefficients could be reordered in phase, using the equations:

$$
\begin{aligned}
& \left(A_{L S B}-r \cos \left(\frac{2 \pi \boldsymbol{\Upsilon}}{N_{S}}\right)\right)^{2}+
\end{aligned}
$$

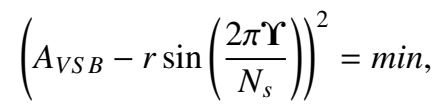

$$
\theta=\frac{2 \pi \Upsilon}{N_{s}} \frac{180}{\pi},
$$

with $r=\sqrt{2 / N_{s}}$. Compared to that reported in Pavia et al. (2018) for the square-back case, a gradual change in the distribution of points can be observed as the chamfer angle is increased. For $\phi_{s}=6^{\circ}$ the points on the $A_{V S B}, A_{L S B}$ plane start to shift towards a new attractor, located in the upper portion of the same plane (at $A_{V S B}>0$, Fig. 11a). This highlights the establishment of a multi-stable condition, that presents some similarities with that reported by Rigas et al. (2014) and Gentile et al. (2016) in the case of axisymmetric bodies. The new attractor eventually becomes the only state for $\phi_{s}=12^{\circ}$ (Fig. $11 \mathrm{~b}$. In this case, the points tend to concentrate in the portion of the scatter plot with $A_{V S B}>0$ and, although there is a higher level of dispersion compared to that seen in previous cases, the two attractors reported for the square-back configuration (Pavia et al. 2018) are no longer visible. All these changes result in noticeable variations in the long-time evolution of the wake, as seen once the phase averaged low order model is considered. The model was constructed using only the time averaged field and the first three POD modes (with the fluctuating term of Eq. 11 ordered in phase and averaged in bins of $15^{\circ}$ ). Two lateral symmetry breaking states are still clearly visible for $\phi_{s}=6^{\circ}$ (Fig. 12). Nevertheless, the reflectional symmetry preserving states seen in Pavia et al. (2018) at $\theta=\pi / 2 \mathrm{rad}$ and $\theta=3 / 2 \pi \mathrm{rad}$ are no longer mirror images of each other. A downwash dominated state is visible for $\theta=\pi / 2 \mathrm{rad}$, that eventually becomes the only stable configuration for $\phi_{s} \geq 12^{\circ}$ (Fig. 13). In these conditions, the wake appears to be locked in a vertical symmetry breaking configuration $(\theta=\pi / 2 \mathrm{rad}$ in Fig. [13, with a swinging motion around this state evident throughout the different phase angles. However, coherence is lost (i.e. the vortical structures burst into smaller eddies) whenever the wake tries to switch to a lateral symmetry breaking state. The transition between a laterally asymmetric bi-stable wake and a stable wake, asymmetric in the vertical direction, is consistent 
with that found by Barros et al. (2017) when perturbing the underbody flow of an Ahmed body with a similar aspect ratio.

Less evident changes are seen in the dynamics of the global oscillating modes reported by Grandemange et al. (2013b), Volpe et al. (2015) and Pavia et al. (2018). The application of tapers to the model vertical trailing edges does not seem to affect the vertical flapping. An inflection point around $S t_{H}=0.20$ is seen in the PSD plot obtained for the temporal coefficients associated with the vertical symmetry breaking mode, for all the chamfer angles considered in the present investigation (Fig. 14b). No variations in either location or amplitude of this point are observed as $\phi_{s}$ is increased. More significant changes are reported for the lateral flapping. As the side shear layers are deflected inwards by the inflow generated by the tapered surfaces and the gap separating them is reduced, stronger aerodynamic interactions are observed between the two sides of the model. This ultimately results in a strengthening of the wake's oscillations in the lateral direction, as highlighted by the growth in amplitude of the peak seen at $S t_{H} \approx 0.17$ in the PSD plot obtained for the temporal coefficient referring to the lateral symmetry breaking POD mode (Fig. 14a). Furthermore, the frequency at which the peak is located is seen to change with the taper angle, shifting from $S t_{H}=0.13$ for the square-back configuration (Volpe et al. (2015) and Pavia et al. (2018)) to $S t_{H}=0.17$ for $\phi_{s} \geq 12^{\circ}$. The latter frequency was also reported by McArthur et al. (2016) when studying the dynamics of the wake downstream of a square-back body with $H>W$. For chamfer angles grater than $12^{\circ}$, this mode becomes so strong that the frequency peak at $S t_{H}=0.17$ becomes the only one visible in the spectrum of the temporal coefficient related to the symmetry preserving mode (Fig. 14c), whereas a small hump can be seen for $\phi_{s} \leq 12^{\circ}$ around at $S t_{H}=0.07$, corresponding to the wake pumping described in Duell and George (1999), Volpe et al. (2015) and Pavia et al. (2018). The peak at $S t_{H}=0.068$ for $\phi_{s}=20^{\circ}$ appears to be different; and is in fact a sub-harmonic of the fan blade passing frequency at a tunnel speed of $40 \mathrm{~m} / \mathrm{s}$ (Baden Fuller, 2012).

\section{Sensitivity of the wake dynamics to small variations of the model pitch angle}

As already discussed in $\$ 3$ the trend observed in the present investigation between the drag of the entire model $\bar{C}_{D}$ and the side taper angle $\phi_{s}$ appears to be in good agreement with that described in the work of Perry et al. (2015) for a similar case. Nevertheless, noticeable differences are observed in the pressure distribution on the model rearward facing surfaces. The pressure maps reported in Perry et al. (2015) for different values of $\phi_{s}$ are all characterised by a positive pressure gradient in

\begin{tabular}{lccc}
$\alpha=0.0^{\circ}$ & $\alpha=-1.0^{\circ}$ & $\alpha=-2.0^{\circ}$ \\
\hline$\partial C_{p} / \partial z^{*}$ & -0.177 & 0.114 & 0.206 \\
\hline
\end{tabular}

Table 1: Vertical pressure gradient for different values of the model pitch angle $\alpha . \partial C_{p} / \partial z^{*}$ was determined considering two taps located on the centreline of the base at $z^{*}=0.490$ and $z^{*}=0.856$.

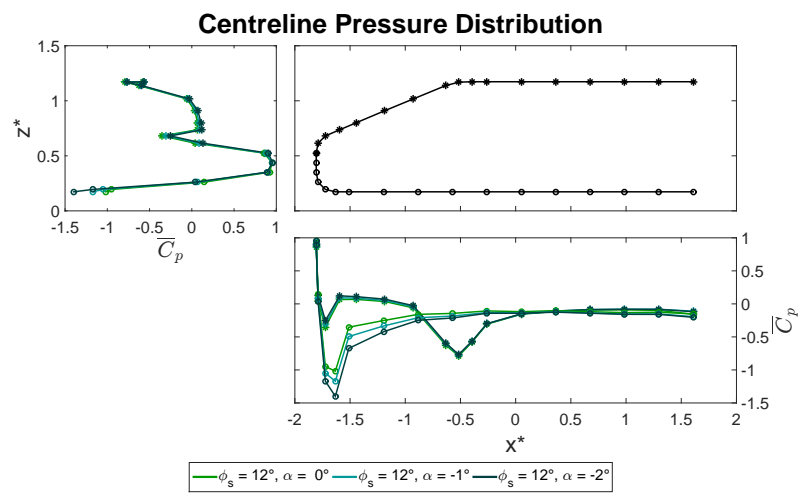

Figure 15: Centreline pressure distribution for the configuration with $\phi_{s}=12^{\circ}$, tested at $\alpha=0.0^{\circ}, \alpha=-1.0^{\circ}$ and $\alpha=-2.0^{\circ}$. The symbol ' $*$ ' denotes the taps located on the upper surfaces whilst ' $\circ$ ' refers to the taps placed on the lower half of the model.

the vertical direction, with the region of lower pressure located close to the bottom trailing edge. This appears to be in contrast with the results obtained here, that show a wake asymmetric in the vertical direction, but with $\frac{\partial C_{p}}{\partial z}<0$ (Fig. 5). To explain the origin of these discrepancies, bearing in mind the high level of sensitivity shown by axisymmetric bodies' wakes to small variations of the pitch angle (Grandemange et al. (2012), Wolf and Stumpf (2014) and Gentile et al. (2017)), an investigation into the effects of small changes of the model pitch angle $\alpha$ on the main time averaged and unsteady features of the wake was carried out. For the sake of simplicity the chamfer angle was fixed at $12^{\circ}$. The model was pitched 'nose down' $\left(\alpha<0^{\circ}\right)$ in order to force the wake to switch from the downwash dominated configuration described in $\$ 3$ to an upwash dominated state. Pitch angles of $-1^{\circ}$ and $-2^{\circ}$ were considered.

During the experiment, the pressure acting on the model base was recorded using a single scanner, as discussed in $\$ 2.4$. A second scanner was employed to determine the pressure distribution on the model centreline. 33 pressure taps were used in this case, with a finer distribution in the locations where the strongest pressure gradients were expected. The time averaged $C_{p}$ data are presented in Fig. 15. The decrease of $\alpha$ is shown to yield a reduction in the static pressure recorded on the model underbody. This is particularly visible at the bottom leading edge of the model's nose, where the suction for $\alpha=0.0^{\circ}$ is further accentuated. The pressure then gradually increases while moving downstream, although the values of $C_{p}$ measured close to the model trailing edges for the pitched configurations are still $\approx 30 \%$ lower than those recorded at $\alpha=0.0^{\circ}$. A pressure increase of similar magnitude is observed on the upper surfaces, further confirming the upwards shift in the location of the front stagnation point. As a result, the pressure difference between the upper and lower surfaces in the region of the model trailing edges is seen to increase. 


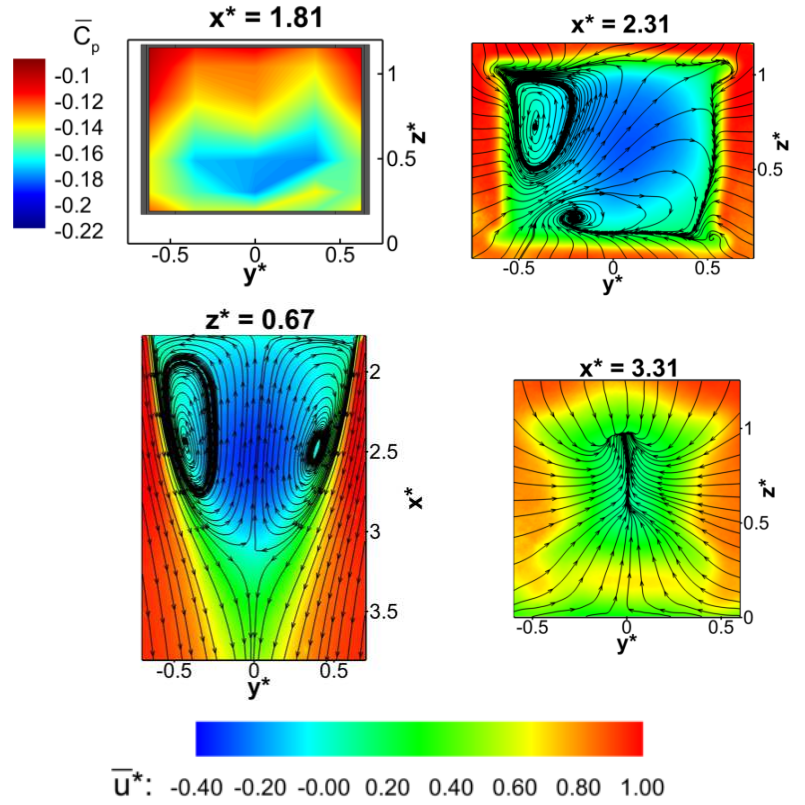

(a) $\phi_{s}=12^{\circ}, \alpha=-1.0^{\circ}$
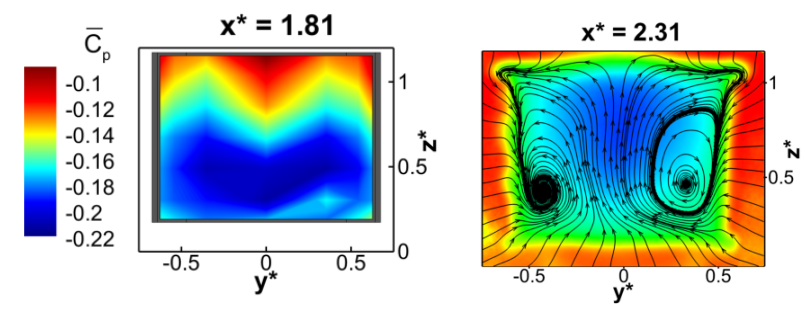

$z^{*}=0.67$
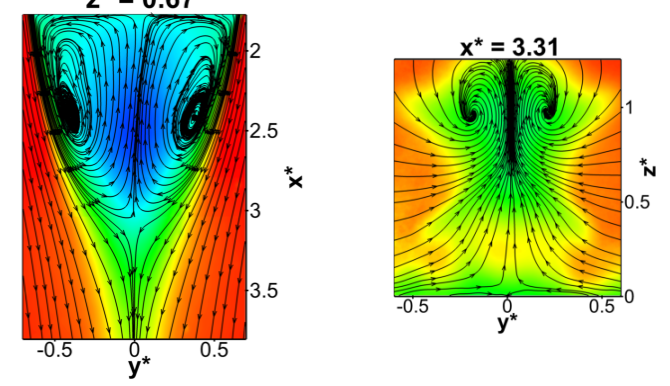

$\bar{u}^{\star}:-0.40-0.20-0.00 \quad 0.20 \quad 0.40 \quad 0.60 \quad 0.80 \quad 1.00$

(b) $\phi_{s}=12^{\circ}, \alpha=-2.0^{\circ}$

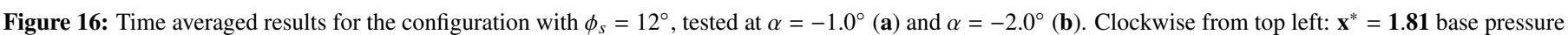
distribution; $\mathbf{x}^{*}=\mathbf{2 . 3 1}$ and $\mathbf{x}^{*}=\mathbf{3 . 3 1}$ PIV stereo cross-planes; $\mathbf{z}^{*}=\mathbf{0 . 6 7}$ PIV horizontal mid-plane. All PIV fields are coloured according to the values of the axial component of the velocity $\overline{u^{*}}$; the streamlines refer to the in-plane components of the velocity.
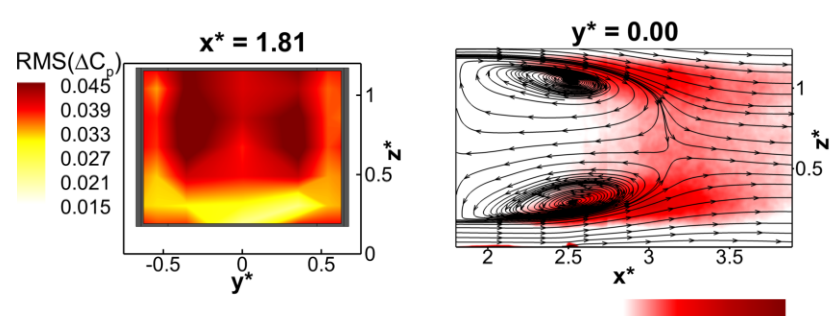

$\mathrm{K}^{*}\left(\mathrm{x} 10^{-3}\right): \quad 01020304050607080$

(a) $\phi_{s}=12^{\circ}, \alpha=-1.0^{\circ}$
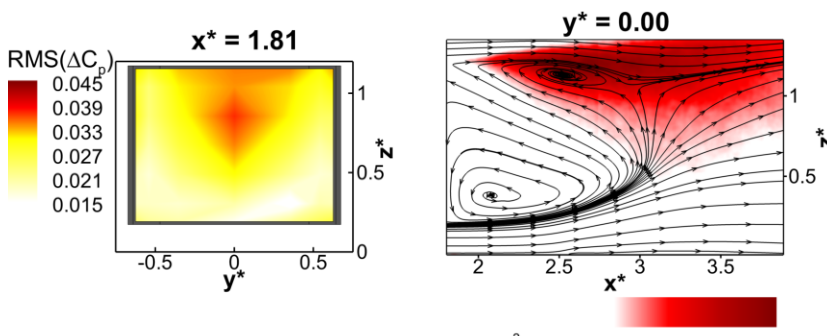

$\mathrm{K}^{*}\left(\times 10^{-3}\right): \quad 01020304050607080$

(b) $\phi_{s}=12^{\circ}, \alpha=-2.0^{\circ}$

Figure 17: Base pressure fluctuations and non dimensional turbulent kinetic energy (at $\left.y^{*}=0.00\right)$ for the configuration with $\phi_{s}=12^{\circ}$, tested at $\alpha=-1.0^{\circ}$, $\alpha=-2.0^{\circ}$ b.

For $\alpha=-1^{\circ}$, a positive pressure gradient is observed on the model base in the vertical direction (Tab. 11). Nevertheless, the magnitude of $\partial C_{p} / \partial z^{*}$ is $\approx 36 \%$ lower than that measured at $\alpha=0.0^{\circ}$, suggesting a more uniform pressure distribution. In these conditions, a $4.7 \%$ drop is observed in the base pressure drag $\left(\bar{C}_{D_{\text {Base }}}\right)$. A region of lower pressure starts to form close to the bottom trailing edge (Fig, 16a first column), matching that shown in the contour plots presented in Perry et al. (2015), and then increases in size and strength when the pitch angle is further decreased (Fig, 16b, first column), resulting in a vertical pressure gradient even stronger in magnitude that that recorded at $\alpha=0^{\circ}$ (Tab. 11). At the same time, $\bar{C}_{D_{\text {Base }}}$ is reported to increase by $8.1 \%$ over the same case.

As previously discussed in $\$ 3$ the changes in pressure distribution observed on the base are the result of modifications in the time averaged wake topology. In particular, the wake is seen to rotate around the centre of the model base as $\alpha$ is de- creased (Fig. 16). No significant variations are observed in the curvature of the side shear layers in the $2 D-2 C$ PIV data at $z^{*}=0.67$.

The symmetry in the vertical direction is almost fully restored for $\alpha=-1.0^{\circ}$. This is particularly evident when looking at the PIV vertical mid-plane (Fig. 17a) and the cross-plane located downstream of the wake closure $\left(x^{*}=3.31\right.$ in Fig. 16a). Something different, however, is seen when the cross-plane located at $x^{*}=2.31$ is considered (Fig. 16a). In this location the wake is aligned along a diagonal running from the bottom-left corner to the top-right corner of the model base. This is similar to that seen in $\$ 3$ for the configuration with the $6^{\circ}$ side edge tapers, although the alignment in that case is with the opposite diagonal. Unlike that observed in $\$ 3$, however, bigger discrepancies are seen when the wake topology captured on this plane is related to the base pressure distribution. From the location of the two vortical structures seen at $x^{*}=2.31$, one would expect 


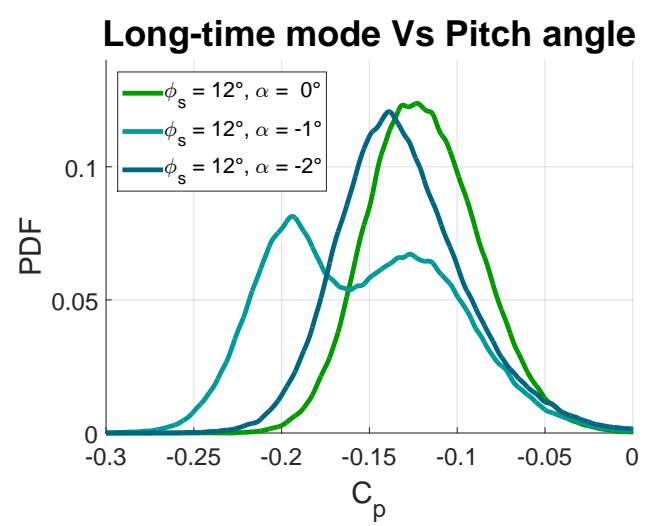

(a)

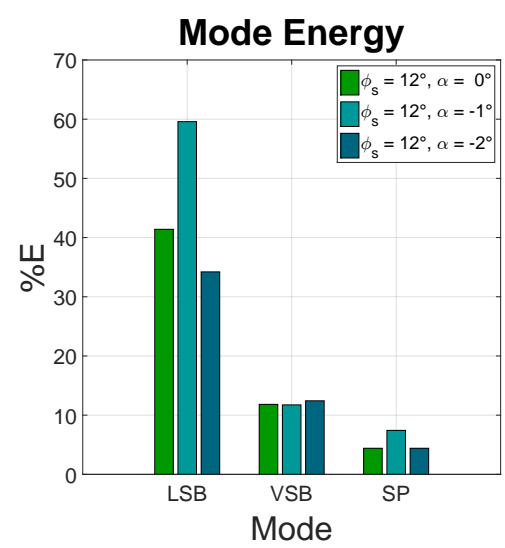

(b)

Figure 18: $\mathrm{a} P \mathrm{PDF}$ distribution of the values of $C_{p}$ recorded by one of the pressure taps placed in the region of highest pressure fluctuation; $\mathbf{b}$ energy associated with the POD modes (base pressure data).
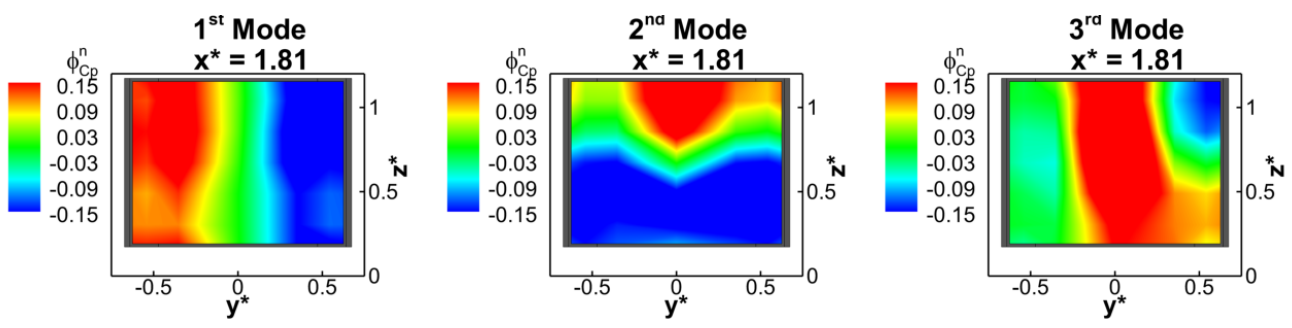

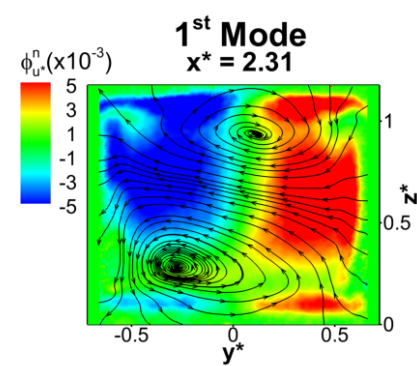

(a) LSB mode

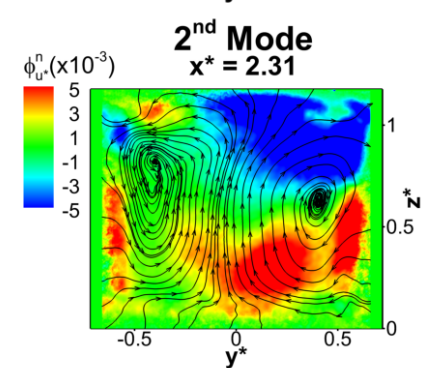

(b) VSB mode

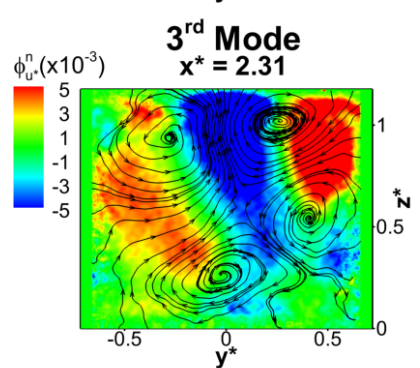

(c) SP mode

Figure 19: Spatial distribution of the POD modes extracted from the base pressure distribution (top row) and PIV cross-plane at $x^{*}=2.31$ (bottom row) for the configuration with $\phi_{s}=12^{\circ}$, tested at $\alpha=-1.0^{\circ}$. The modes are ordered according to their topology: a lateral symmetry breaking mode ( $\left.L S B\right)$, $\mathbf{b}$ vertical symmetry breaking mode $(V S B)$, c symmetry preserving mode $(S P) . \phi_{C_{p}}^{n}$ refers to the magnitude of the spatial eigen-modes extracted from the field of the pressure fluctuation. The eigen-functions related to the velocity fluctuation are coloured according to the values of the through plane component $\phi_{u^{*}}^{n}$ whereas the streamlines are drawn considering the in-plane components $\phi_{v^{*}}^{n}$ and $\phi_{w^{*}}^{n}$.

a larger suction on the left-hand side of the base and a better pressure recovery on the opposite side. Instead, a good level of lateral symmetry is observed in the pressure map reported in Fig. 16a. The reason for these differences is that the duration of the PIV recordings is $T_{\text {samp }} \approx 137 \mathrm{~s}$, compared to $T_{\text {samp }}=630 \mathrm{~s}$ for the pressure tapping acquisition, and, with an average time between switches of over $5 s$, the results have been biased towards one state. In addition, as a bi-stable or multi-stable condition is approached, the effect of small misalignments in the experimental setup tends to be amplified, increasing the occurrence of a particular state. This has already been observed in the case of variations of the yaw angle (Volpe et al. (2015),
Evrard et al. (2016), Pavia et al. (2018)), and, in analogy with that seen in the case of axisymmetric bodies by Gentile et al. (2017), seems also to apply to changes in the pitch angle.

The long-time instability, that almost completely disappears when $12^{\circ}$ side tapers are applied to the model at $0^{\circ}$ pitch (see $\$ 4$, gains strength when the model pitch angle is changed to $\alpha=-1.0^{\circ}$ and the symmetry in the vertical direction in the time averaged wake topology is recovered. This becomes clear when looking at the unsteady results presented in Fig. 17a As the symmetry in the vertical direction is restored, a more even distribution of turbulent kinetic energy can be observed 

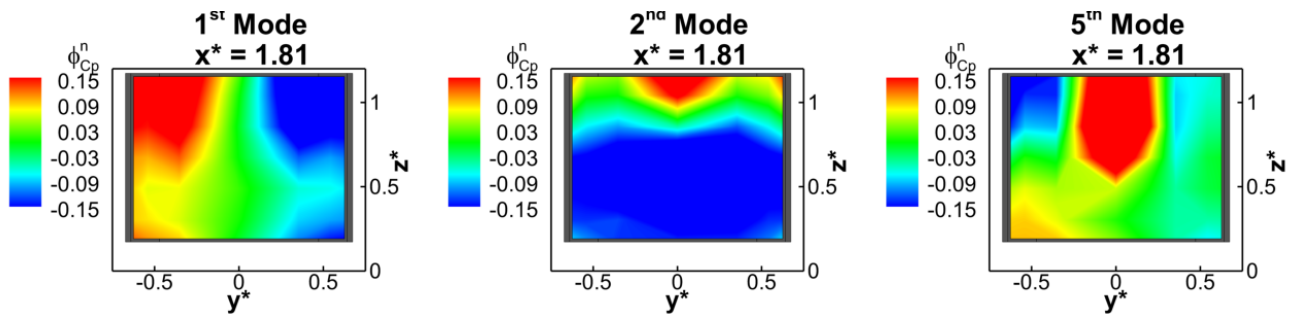

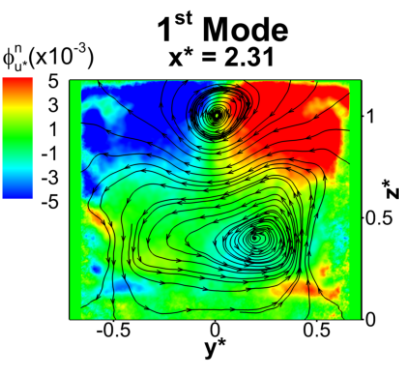

(a) LSB mode

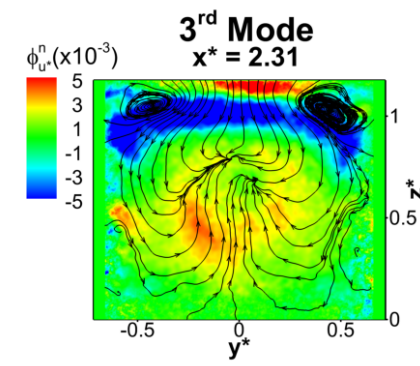

(b) VSB mode

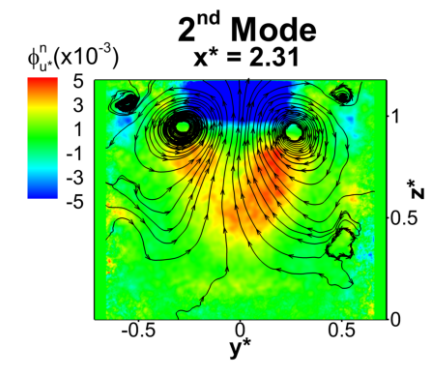

(c) SP mode

Figure 20: Spatial distribution of the POD modes extracted from the base pressure distribution (top row) and PIV cross-plane at $x^{*}=2.31$ (bottom row) for the configuration with $\phi_{s}=12^{\circ}$, tested at $\alpha=-2.0^{\circ}$. The modes are ordered according to their topology: a lateral symmetry breaking mode $(L S B)$, $\mathbf{b}$ vertical symmetry breaking mode $(V S B)$, c symmetry preserving mode $(S P) . \phi_{C_{p}}^{n}$ refers to the magnitude of the spatial eigen-modes extracted from the field of the pressure fluctuation. The eigen-functions related to the velocity fluctuation are coloured according to the values of the through plane component $\phi_{u^{*}}^{n}$ whereas the streamlines are drawn considering the in-plane components $\phi_{v^{*}}^{n}$ and $\phi_{w^{*}}^{n}$.



(a)

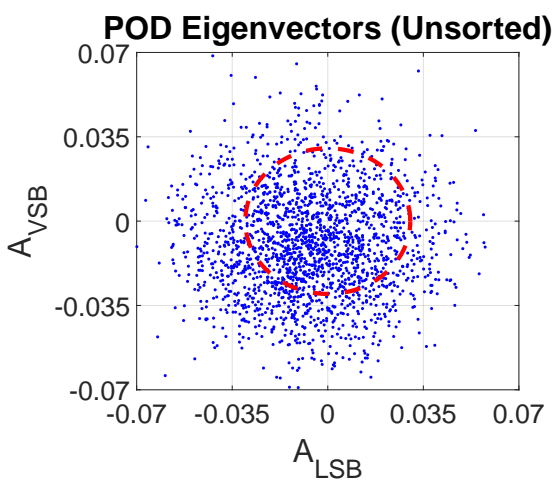

(b)

Figure 21: Scatter plots of the POD temporal coefficients associated with the $L S B$ mode and the $V S B$ mode for the configuration with $\phi_{S}=12^{\circ}$, tested at $\alpha=-1.0^{\circ}$ a a and $\alpha=-2.0^{\circ}$ [b. The dashed circle represents the fitting function used for sorting the snapshots in phase (see Eq. 17). Data referring to the PIV cross-plane recorded at $x^{*}=2.31$.

between the two horizontal shear layers. Indeed, having a similar amount of turbulent activity between two opposite shear layers seems to be a necessary condition for the wake symmetry. An increase in the level of unsteadiness can also be noticed on the base. The region of high pressure fluctuation is reported to increase in size and the highest values of $\operatorname{RMS}\left(\Delta C_{p}\right)$ tend to cluster around two lobes, similar to that seen in the case of bi-stable wakes. A larger scatter is observed in the values of $C_{p}$ recorded in this region. Indeed, as shown in Fig. 18a, the PDF of the signal recorded by one of the taps located in this area tends towards a bi-modal distribution, similar to that reported in Pavia et al. (2016) for the square-back case. But the similarities with the square-back configuration go further than that. Strong analogies between this case and that studied in Pavia et al. (2018) are seen in the distribution of the fluctuat- ing energy between the first three modes (Fig. 18), with the lateral symmetry breaking mode alone accounting now for at least $60 \%$ of the energy, and in the shape of the spatial functions associated with the modes themselves. As shown in Fig. 19a and 19b the first two orthogonal symmetry breaking modes extend for the entirety of the base as well as the wake crosssection measured at $x^{*}=2.31$, further confirming the analogies with the square-back case investigated in Pavia et al. (2018). Similar considerations apply to the symmetry preserving mode, whose energy level is more than $60 \%$ higher than that reported at $\alpha=0.0^{\circ}$ (Fig. 18b). Furthermore, two planes of symmetry can be seen in the spatial function related to this mode when the PIV cross-plane located at $x^{*}=2.31$ is considered (second row in Fig. 19c). Unlike that seen in Pavia et al. (2018), however, the two planes are not aligned with the two symmetry planes 

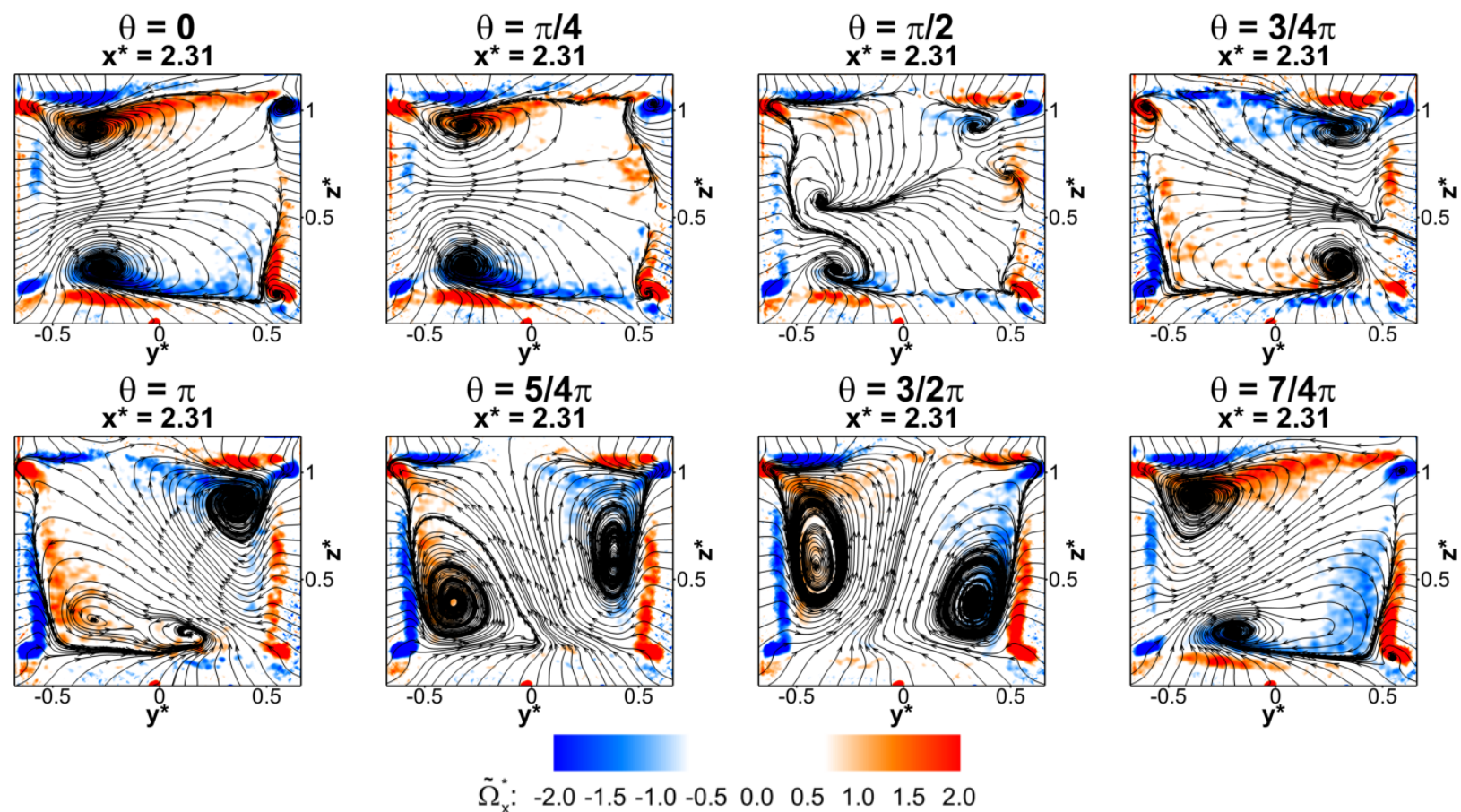

of the base but form an angle of $\approx 45^{\circ}$. This may be related to the fact that in this case the time averaged wake appears to be oriented diagonally.

The re-activation of the long-time instability is accompanied with the appearance of multiple states. This is clear when look- ing at the scatter plot between the temporal eigenvectors related to the two symmetry breaking modes, reported in Fig. 21a Unlike that seen for the model tested at $\alpha=0.0^{\circ}$ (Fig. 11b), the points on the $A_{V S B}, A_{L S B}$ plane tend now to cluster around multiple attractors, resulting in a ' $U$ ' shaped cloud similar to
838 839 840 841 842

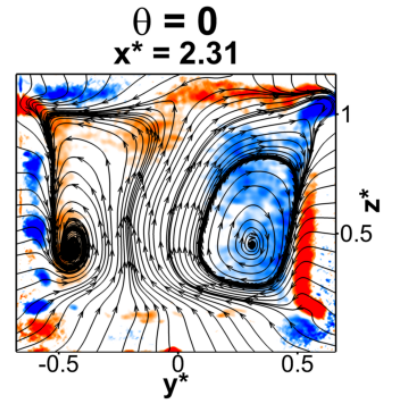

$\theta=\pi$

$\mathrm{x}^{*}=\mathbf{2 . 3 1}$

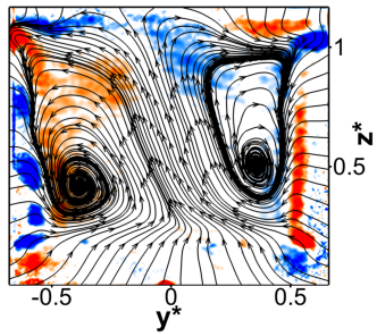

$\theta=\pi / 4$

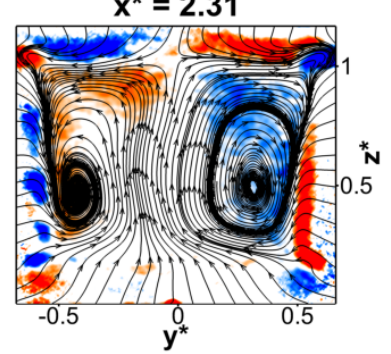

$\theta=5 / 4 \pi$

$\mathbf{x}^{*}=\mathbf{2 . 3 1}$
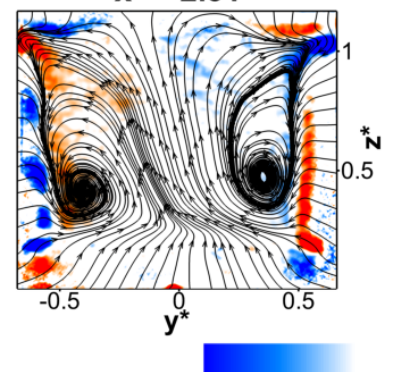

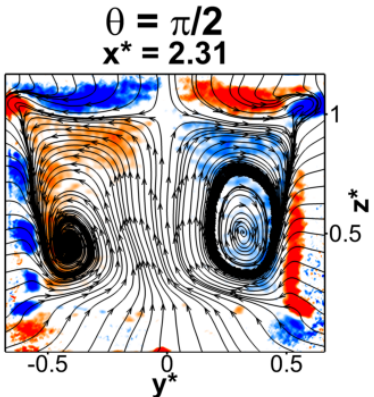

$\theta=3 / 2 \pi$

$\mathrm{x}^{*}=2.31$

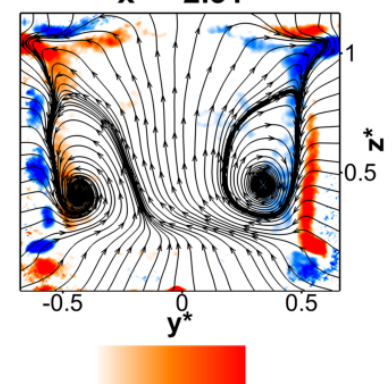

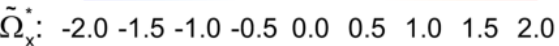

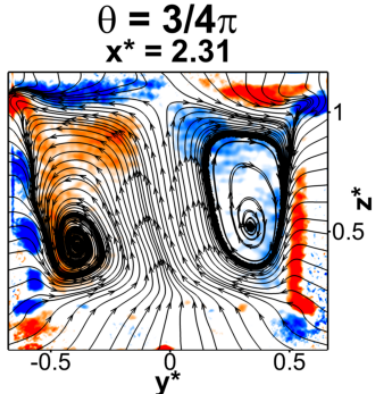

$\theta=7 / 4 \pi$

$\mathrm{x}^{*}=\mathbf{2 . 3 1}$

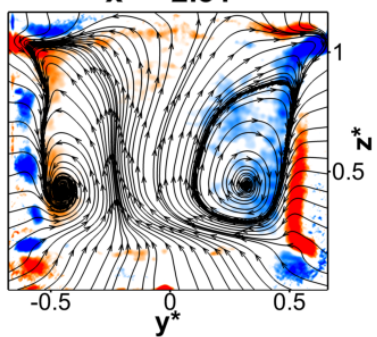

Figure 23: Low order phase averaged velocity field at $x^{*}=2.31$ for the configuration with $\phi_{s}=12^{\circ}$, tested at $\alpha=-2.0^{\circ}$. The plots are coloured according to the values of the normalised streamwise component of the vorticity $\tilde{\Omega}_{x}^{*}$; the streamlines are drawn considering the in-plane components of the velocity field. 

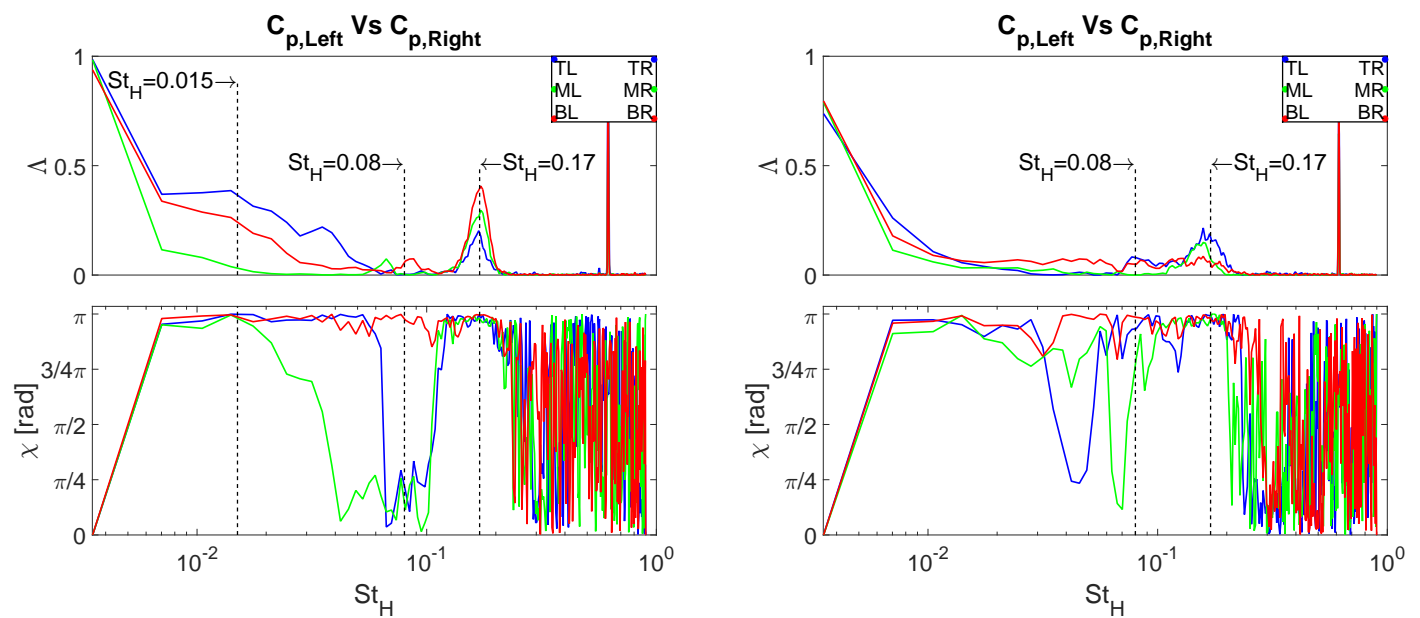

(a) $\alpha=0.0^{\circ}$

(b) $\alpha=-1.0^{\circ}$
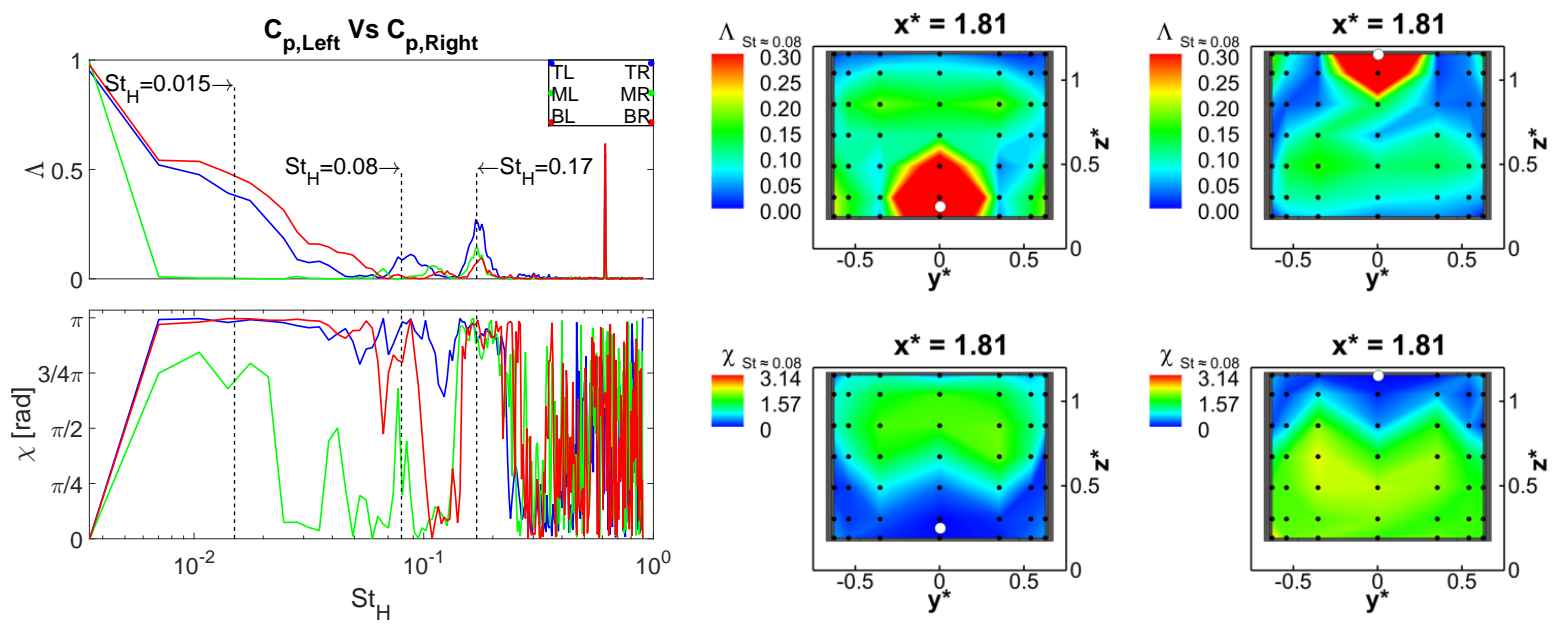

(c) $\alpha=-2.0^{\circ}$

(d) $\alpha=0.0^{\circ}$

(e) $\alpha=-2.0^{\circ}$

Figure 24: $\mathbf{a}$ b $\quad$ c two-point coherence analysis performed considering the unsteady signal recorded by pressure taps placed at different locations along the vertical trailing edges of the model base, for the configuration with $\phi_{s}=12^{\circ}$ tested at different pitch angles: $\mathbf{a} \alpha=0.0^{\circ} ; \mathbf{b} \alpha=-1.0^{\circ} ; \mathbf{c} \alpha=-2.0^{\circ}$. $\mathbf{d}$ e contour maps showing the coherence magnitude and phase between the signal recorded by the tap closest to the rear stagnation point and all the remaining taps on the base at $S t_{H} \approx 0.07$ for $\Theta=0.0^{\circ}$ (d) and $\Theta=-2.0^{\circ}$ (e).

that seen for the configuration equipped with $6^{\circ}$ side edge tapers (Fig. 11a), apart from the fact that the third attractor is now located in the lower half of the plot $\left(A_{V S B}<0\right)$ rather than being in the upper portion $\left(A_{V S B}>0\right)$. The wake now switches between two lateral symmetry breaking states and a vertical symmetry breaking state, as shown in the phase averaged low order model reported in Fig. 22, constructed using the dataset recorded at $x^{*}=2.31$ following the procedure described in $\$ 4$ Another difference with the multi-stable case discussed in $\$ 4$ is the fact that the vertical symmetry breaking state (now observed at $\theta=3 / 2 \pi$ ) appears to be upwash dominated rather than downwash dominated. This is linked to the different distributions seen in the scatter plot on the $A_{V S B}, A_{L S B}$ plane between the these two cases. From the plots presented in Fig. 22, it can also be noticed that only one symmetry preserving state is retained $(\theta=\pi / 2$ in Fig. 22). As pointed out in previous cases, this state is still characterised by the presence of four recirculating structures. These structures, however, are now diagonally aligned, spanning from the bottom-left corner to the top-right corner of the base, following the same orientation seen for the symmetry preserving mode.

From that seen so far, it can be inferred that the reduction in base drag observed at $\alpha=-1.0^{\circ}$ has to be ascribed mainly to the symmetrisation of the time averaged wake rather than being a consequence of the suppression of the long-time instability, which in fact appears to be much stronger than that seen at $\alpha=0.0^{\circ}$.

The upwash dominated, vertical symmetry breaking state becomes eventually the only state when the pitch angle of the model is decreased to $\alpha=-2.0^{\circ}$ (Fig. 23). In these conditions, the wake locks into a stable state, which appears to be the mirror image of that depicted in Fig. 7d for the same configu- 


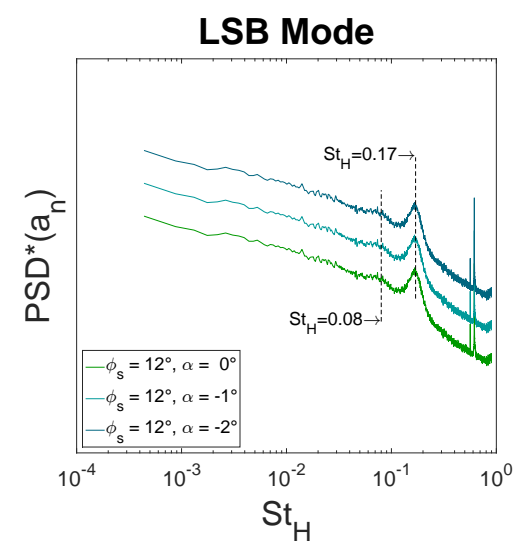

(a)

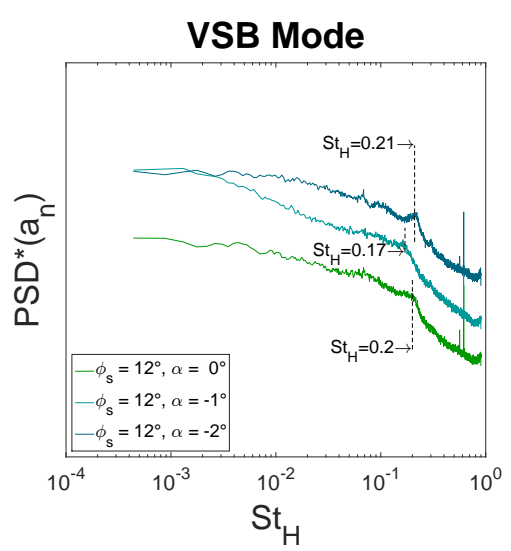

(b)

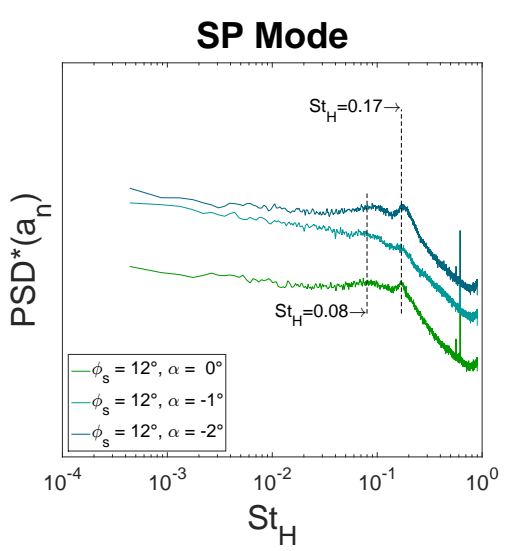

(c)

Figure 25: Spectra of the POD temporal coefficients associated with the lateral symmetry breaking mode ( $L S B$, a), the vertical symmetry breaking mode (VS $B$, $[\mathbf{b}$, the symmetry preserving mode $(S P, \mathbf{c})$. The curves have been shifted along the vertical axis.

ration tested at $\alpha=0.0^{\circ}$. The level of unsteadiness seen in the pressure field recorded on the model base, as well as in the velocity field measured at $y^{*}=0.00$, is greatly reduced compared to that seen for $\alpha=-1.0^{\circ}$. Fluctuations are now limited to the region around the top shear layer (Fig. 17b). This is the only portion of the flow field where coherent motions in the lateral $(L S B)$, vertical $(V S B)$ and longitudinal $(S P)$ directions can still be seen (Fig. 20). A similar trend was reported by Gentile et al. (2017) when changing the pitch angle of an axisymmetric body with a blunt trailing edge. The disappearance of the long-time instability is accompanied with a noticeable reduction of the amount of fluctuating energy captured by the $L S B$ mode. Indeed, as observed in the plot in Fig. 18b the energy associated with the lateral symmetry breaking mode in this case drops to a level even lower than that obtained for the downwash dominated stable wake observed for $\alpha=0.0^{\circ}$. Further confirmations of the absence of a multi-stable condition are given by the shape of the PDF of $C_{p}$ in the region of the base characterised by the highest level of fluctuation (Fig. 18a), and the increased level of scattering observed among the points in the $A_{V S B}, A_{L S B}$ plane (Fig. 21b). Nevertheless, an attractor located at $A_{V S B}<0$, corresponding to the upwash dominated state observed in the phase averaged low order model reported in Fig. 23 , is persistent and the motion of the wake is reduced to limited variations in the orientation of the flow reversal, as it oscillates from the top-left corner to the top-right corner of the base. These oscillations may be linked with the hump seen at $S t_{H} \approx 0.015$ in the plots referring to the magnitude of the two-point coherence analysis performed between the top-left (TL) and top-right (TR) taps as well as the bottom-left (BL) and bottom-right (BR) taps, following the procedure described in $\$ 2.4$ (Fig. 24c). The $\pi \mathrm{rad}$ phase angle observed for this hump seems to further support this thesis.

An inflection point at a similar frequency is also seen for the case with $\alpha=0.0^{\circ}$ (Fig. 24a), but it disappears for $\alpha=-1.0^{\circ}$, when the long-time instability, characterised by random switches between states, takes place (Fig. 24b). As far as the global oscillating modes are concerned, the unsteady behaviour of the wake is dominated by the lateral flapping at $S t_{H}=0.17$. This is evident when considering the plots referring to the two-point coherence analysis presented in Fig. 24 as well as the PSD of the POD temporal coefficients presented in Fig. 25 The 'strength' of this motion, however, appears to change along the vertical direction depending on the orientation of the wake, matching the changes seen in the spatial function related to the $L S B$ mode reported in Fig. 10a and 20a Indeed, the highest peak, in terms of coherence magnitude recorded at $S t_{H}=0.17$, is seen between the lower pairs of taps in the case of a downwash dominated wake ( $\alpha=0.0^{\circ}$, Fig. 24a), but then changes to the upper pair of taps for an upwash dominated wake ( $\alpha=-2.0^{\circ}$, Fig. 24c), following the stabilisation of the upper and lower recirculation respectively. A similar trend is also observed for the peak located at $S t_{H}=0.08$ and associated with the 'wake pumping' (Duell and George 1999). Unlike that seen in Pavia et al. (2018) in the case of laterally bi-stable wakes, however, the mode associated with this frequency appears to be linked with a swinging motion of the horseshoe vortex depicted in Fig. 7d around the rear stagnation point, rather than being the result of an alternated 'stretching' and 'squeezing' in the streamwise direction alone. The coherence analysis performed at $S t_{H} \approx 0.08$ between one of the taps closest to the rear stagnation point and all the remaining pressure sensors placed on the base shows indeed the presence of a large region with relatively good coherence $(\Lambda>0.1)$ and a phase angle $\chi(f) \approx \pi / 2 \mathrm{rad}$, which seems to be compatible with the existence of such a motion. This is observed at $\alpha=0.0^{\circ}$ (Fig. 24d) as well as $\alpha=-2.0^{\circ}$ (Fig. 24e).

A different trend is observed for the non-dimensional frequency associated with the vertical flapping. The PSD plot obtained for the temporal coefficient related to the vertical symmetry breaking POD mode (Fig. 25b), shows that the frequency characteristic of this motion drops from $S t_{H}=0.20$ to $S t_{H}=0.17$ when the symmetry in the vertical direction is recovered in the wake and the gap separating the top and bottom shear layers is widened (at $\alpha=-1.0^{\circ}$ ), then increases to $S t_{H}=0.21$ when the pitch angle of the model is changed to 
$\alpha=-2.0^{\circ}$ and an upwash dominated wake is formed.

\section{Summary and conclusions}

In this paper, the aerodynamic effects produced by high aspect ratio tapers applied to the side trailing edges of a simplified square-back body without wheels have been investigated. The tapered surfaces have been shown to trigger a switch from a laterally asymmetric bi-stable wake to a stable wake, asymmetric in the vertical direction. The wake has been observed to retain a topology similar to that described in Pavia et al. (2018) for the lateral symmetry breaking states downstream of the simple square-back configuration (with no tapers), although rotated by $90^{\circ}$. As the chamfer angle $\phi_{s}$ is increased, the horizontal pressure gradient seen in the case of lateral symmetry breaking states is replaced by a (negative) vertical pressure gradient, being the result of the formation of a downwash dominated wake.

$\mathrm{A} \approx 6 \%$ drag reduction compared to the square-back case is reported for taper angles between $6^{\circ}$ and $12^{\circ}$. This gain is ascribed to the circular vortex, responsible for the suction zone visible in any symmetry breaking state, that tends to 'stretch' in the streamwise direction, resulting in a $\approx 15 \%$ reduction of the base pressure drag. No particular changes are observed in the short-time wake dynamics, except from a noticeable strengthening of the lateral flapping motion, as a consequence of the higher level of interactions between the two lateral shear layers, and a weakening of the pumping motion as $\phi_{s}$ is increased.

A better understanding of the transition from a laterally asymmetric bi-stable wake to a stable wake, asymmetric in the vertical direction, has been achieved by applying the phase averaged low order model already used in Pavia et al. (2018). For $\phi_{s}=6^{\circ}$, the wake has been observed to switch between two lateral symmetry breaking states and a vertical symmetry breaking state, resulting in a multi-stable condition that presents some similarities with that reported by Rigas et al. (2014) and Gentile et al. (2016) in the case of axisymmetric bodies. The latter state eventually becomes the only stable configuration of the wake for $\phi_{s} \geq 12^{\circ}$. In these conditions the wake loses coherence every time the vortical structures come closer to the side shear layer. The long-time instability is replaced by a swinging motion around the rear stagnation point, with a characteristic frequency of $S t_{H} \approx 0.015$. A similar change is seen in the mode associated with frequency peak located at $S t_{H}=0.08$.

The transition between these two scenarios is consistent with that found by Barros et al. (2017) when perturbing the underbody flow of a similarly shaped body. The fact that a similar behaviour can be obtained regardless of the shear layer to which the perturbation is applied, suggests that the long-time instability is indeed the result of the establishment of a condition of equilibrium among all four shear layers bounding the wake. This may also explain the link between the orientation of the long-time symmetry breaking mode and the model aspect ratio found by Grandemange et al. (2013a). The fact that the wake is seen to switch laterally for $W>H$ and vertically for $W<H$ may be ascribed to the existence of stronger interactions between the horizontal shear layers in the first case and the vertical shear layers in the second case. This trend is consistent with that seen for the global oscillating modes, with a lateral flapping stronger than the vertical flapping when the gap between the vertical shear layers is less than that one separating the horizontal shear layers.

A further confirmation of the fact that the long-time instability is the result of a condition of global equilibrium, and not just a function of the state of perturbation of a single shear layer, has been obtained by studying the sensitivity of the wake to small variations of the model pitch angle. A strengthening of the long-time instability is evident every time the symmetry in the vertical direction in the time averaged wake is recovered. In the same conditions, a further reduction of the base drag over the same model tested at zero degree pitch has also been observed (with $\Delta \bar{C}_{D_{\text {Base }}}=-4.9 \%$ ). This is in good agreement with the findings of Grandemange et al. (2015) and seems to suggest that a lower drag wake is not necessarily a more stable wake. Furthermore, a pitch angle variation of $\approx-1^{\circ}$ has been found to be sufficient to force the time averaged wake to switch from a downwash dominated topology to an upwash dominated topology. This high level of sensitivity may explain the differences when comparing the time averaged results obtained in the present investigation with those reported in the literature (Perry et al., 2015). The perturbation applied to the model in this case, in fact, is much weaker than that used for example in Castelain et al. (2018) to trigger a similar switch, further confirming the existence of a delicate equilibrium among all shear layers that may also be at the root of the high level of variability seen in the wake topologies reported by Makihara et al. (2016) when considering more realistic vehicle shapes.

\section{Acknowledgements}

The authors would like to thank Jaguar Land Rover for the financial support. Thanks are also due to Mr. David Cooper, Mr. Nigel Lines and Mr. Andrew Horsey, for their excellent work in manufacturing the models and keeping the test facility always in optimal conditions.

\section{References}

Ahmed, S., Ramm, G., and Faitin, G. (1984). Some salient features of the timeaveraged ground vehicle wake. Technical report, Society of Automotive Engineers, Inc., Warrendale, PA.

Baden Fuller, J. (2012). The unsteady aerodynamics of static and oscillating simple automotive bodies. $\mathrm{PhD}$ thesis, Loughborough University.

Barros, D., Borée, J., Cadot, O., Spohn, A., and Noack, B. R. (2017). Forcing symmetry exchanges and flow reversals in turbulent wakes. Journal of Fluid Mechanics, 829.

Benedict, L. and Gould, R. (1996). Towards better uncertainty estimates for turbulence statistics. Experiments in fluids, 22(2):129-136.

Bonnavion, G., Cadot, O., Évrard, A., Herbert, V., Parpais, S., Vigneron, R., and Délery, J. (2017). On multistabilities of real car's wake. Journal of Wind Engineering and Industrial Aerodynamics, 164:22-33.

Brackston, R., de la Cruz, J. G., Wynn, A., Rigas, G., and Morrison, J. (2016). Stochastic modelling and feedback control of bistability in a turbulent bluff body wake. Journal of Fluid Mechanics, 802:726-749.

Cabitza, S. (2014). Active control of the wake from a rectangular-sectioned body. $\mathrm{PhD}$ thesis, $\mathrm{PhD}$ thesis. Imperial College London. 
Castelain, T., Michard, M., Szmigiel, M., Chacaton, D., and Juvé, D. (2018). Identification of flow classes in the wake of a simplified truck model depending on the underbody velocity. Journal of Wind Engineering and Industrial Aerodynamics, 175:352-363.

Duell, E. G. and George, A. (1999). Experimental study of a ground vehicle body unsteady near wake. Technical report, SAE Technical Paper.

Evrard, A., Cadot, O., Herbert, V., Ricot, D., Vigneron, R., and Délery, J. (2016). Fluid force and symmetry breaking modes of a 3d bluff body with a base cavity. Journal of Fluids and Structures, 61:99-114.

Gentile, V., Schrijer, F., Van Oudheusden, B., and Scarano, F. (2016). Lowfrequency behavior of the turbulent axisymmetric near-wake. Physics of Fluids, 28(6):065102.

Gentile, V., Van Oudheusden, B., Schrijer, F., and Scarano, F. (2017). The effect of angular misalignment on low-frequency axisymmetric wake instability. Journal of Fluid Mechanics, 813.

Grandemange, M. (2013). Analysis and control of three-dimensional turbulent wakes: from axisymmetric bodies to road vehicles. PhD thesis, Palaiseau, Ecole polytechnique.

Grandemange, M., Cadot, O., Courbois, A., Herbert, V., Ricot, D., Ruiz, T. and Vigneron, R. (2015). A study of wake effects on the drag of ahmed s squareback model at the industrial scale. Journal of Wind Engineering and Industrial Aerodynamics, 145:282-291.

Grandemange, M., Gohlke, M., and Cadot, O. (2013a). Bi-stability in the turbulent wake past parallelepiped bodies with various aspect ratios and wall effects. Physics of Fluids (1994-present), 25(9):95-103.

Grandemange, M., Gohlke, M., and Cadot, O. (2013b). Turbulent wake past a three-dimensional blunt body. part 1. global modes and bi-stability. Journal of Fluid Mechanics, 722:51-84.

Grandemange, M., Gohlke, M., and Cadot, O. (2014a). Statistical axisymmetry of the turbulent sphere wake. Experiments in fluids, 55(11):1838.

Grandemange, M., Gohlke, M., and Cadot, O. (2014b). Turbulent wake past a three-dimensional blunt body. part 2. experimental sensitivity analysis. Journal of Fluid Mechanics, 752:439-461.

Grandemange, M., Gohlke, M., Parezanović, V., and Cadot, O. (2012). On experimental sensitivity analysis of the turbulent wake from an axisymmetric blunt trailing edge. Physics of fluids, 24(3):035106.

Johl, G. (2010). The design and performance of a $1.9 \mathrm{mx} 1.3 \mathrm{~m}$ indraft wind tunnel. PhD thesis, (c) Guru Johl.

Krajnovic, S. and Davidson, L. (2003). Numerical study of the flow around a bus-shaped body. Journal of Fluids Engineering, 125(3):500-509.

Lumley, J. L. (1967). The structure of inhomogeneous turbulent flows. Atmospheric turbulence and radio wave propagation, pages 166-178.

Makihara, T., Kitamura, T., Yamashita, T., Maeda, K., Kato, C., Takayama, T., Yamamoto, K., Yamade, Y., and Suzuki, Y. (2016). Identification of vortical structure that drastically worsens aerodynamic drag on a 2 box vehicle using large-scale simulations. SAE International Journal of Passenger Cars-Mechanical Systems, 9(2016-01-1585).

Mariotti, A. (2018). Axisymmetric bodies with fixed and free separation: Basepressure and near-wake fluctuations. Journal of Wind Engineering and Industrial Aerodynamics, 176:21-31.

McArthur, D., Burton, D., Thompson, M., and Sheridan, J. (2016). On the near wake of a simplified heavy vehicle. Journal of Fluids and Structures, 66:293-314.

Pavia, G. and Passmore, M. (2017). Characterisation of wake bi-stability for a square-back geometry with rotating wheels. In FKFS Conference, pages 93-109. Springer.

Pavia, G., Passmore, M., and Gaylard, A. (2016). Influence of short rear end tapers on the unsteady base pressure of a simplified ground vehicle. Technical report, SAE Technical Paper.

Pavia, G., Passmore, M., and Sardu, C. (2018). Evolution of the bi-stable wake of a square-back automotive shape. Experiments in Fluids, 59(1):20.

Perry, A. K. (2016). An investigation into the base pressure of simplified automotive squareback geometries. $\mathrm{PhD}$ thesis, Loughborough University.

Perry, A.-K., Passmore, M., and Finney, A. (2015). Influence of short rear end tapers on the base pressure of a simplified vehicle. SAE International Journal of Passenger Cars-Mechanical Systems, 8(2015-01-1560):317327.

Perry, A.-K., Pavia, G., and Passmore, M. (2016). Influence of short rear end tapers on the wake of a simplified square-back vehicle: wake topology and rear drag. Experiments in Fluids, 57(11):169.

Prasad, A. K. (2000). Stereoscopic particle image velocimetry. Experiments in fluids, 29(2):103-116.

Rigas, G., Oxlade, A., Morgans, A., and Morrison, J. (2014). Low-dimensional dynamics of a turbulent axisymmetric wake. Journal of Fluid Mechanics, 755:R5.

SAE (2010). Surface vehicle recommended practice. Technical Report J1594, SAE International.

Sims-Williams, D. B. and Dominy, R. (1998). Experimental investigation into unsteadiness and instability in passenger car aerodynamics. Technical report, SAE Technical Paper.

Sirovich, L. (1987). Turbulence and the dynamics of coherent structures. part i: Coherent structures. Quarterly of applied mathematics, 45(3):561-571.

Taira, K., Brunton, S. L., Dawson, S. T., Rowley, C. W., Colonius, T., McKeon, B. J., Schmidt, O. T., Gordeyev, S., Theofilis, V., and Ukeiley, L. S. (2017). Modal analysis of fluid flows: An overview. AIAA Journal, pages $1-29$.

Taneda, S. (1978). Visual observations of the flow past a sphere at reynolds numbers between 104 and 10 6. Journal of Fluid Mechanics, 85(1):187192.

Van Raemdonck, G. and Van Tooren, M. (2008). Time averaged phenomenological investigation of a wake behind a bluff body. In Bluff Body Aerodynamics and Application VI Conference, Milan, Italy.

Volpe, R., Devinant, P., and Kourta, A. (2015). Experimental characterization of the unsteady natural wake of the full-scale square back ahmed body: flow bi-stability and spectral analysis. Experiments in Fluids, 56(5):1-22.

Willert, C. E. and Gharib, M. (1991). Digital particle image velocimetry. Experiments in fluids, 10(4):181-193.

Wolf, C. C. and Stumpf, E. (2014). The subsonic near wake of bluff bodies. $\mathrm{PhD}$ thesis, Lehrstuhl und Institut für Luft-und Raumfahrtsysteme (ILR).

Wood, D. (2015). The effect of rear geometry changes on the notchback flow field. $\mathrm{PhD}$ thesis, Loughborough University. 